\title{
Complete spectra of the delayed luminescence from aromatic compounds in liquid solutions: on the observability of direct radiative triplet-triplet annihilation
}

\author{
Bernhard Nickel ${ }^{1}$ and Hans-Josef Karbach ${ }^{2}$ \\ Max-Planck-Institut für Biophysikalische Chemie, Abteilung Spektroskopie, Am Fassberg, D-3400 Göttingen, FRG
}

Received 28 February 1990

Dedicated to Professor Albert Weller on the occasion of his retirement

\begin{abstract}
With many aromatic compounds in liquid solution, the population of the metastable triplet state $T_{1}$ leads to delayed $S_{n} \rightarrow S_{0}$ fluorescence (DF $n, n \geqslant 1$ ) of the pyrene type, which results from the generation of excited singlet states $S_{n}$ by nonradiative triplettriplet annihilation (TTA) $T_{1}+T_{1} \rightarrow S_{n}+S_{0}$. Knox and Swenberg have postulated that, apart from the DFl, there should be an extremely weak direct radiative TTA: $T_{1}+T_{1} \rightarrow S_{0}+S_{0}+h \nu_{\text {rad. }}$. The spectrum of the direct radiative TTA is likely to be similar to the convolution of the phosphorescence spectrum with itself. Due to this similarity, part of the high-energy delayed luminescence from solutions of pyrene and phenazine can be assigned to direct radiative TTA. The probability of direct radiative TTA relative to the generation of $S_{n}$ by nonradiative TTA is typically $\$ 5 \times 10^{-7}$. With anthracene, 9-methylanthracene and 1,2-benzanthracene no similarity has been found between the spectrum of the observed high-energy luminescence and the calculated spectrum of radiative TTA. Some special aspects of the delayed fluorescence are treated: nonradiative TTA as a special case of Dexter energy transfer, the temperature dependence of the delayed excimer fluorescence from pyrene, nonequilibrium hot-band delayed fluorescence from $S_{1}$ of anthracene, and the problem of the indirect determination of lifetimes of upper excited singlet states.
\end{abstract}

\section{Introduction}

With many aromatic compounds, electronically excited singlet states $S_{n}(n \geqslant 1)$ are generated by the bimolecular reaction of molecules in the metastable triplet state $T_{1}$, and a delayed fluorescence from $S_{n}$ (DF $n, n \geqslant 1$ ) can be observed [1,2]:

$\mathrm{T}_{1}+\mathrm{T}_{1} \leftrightarrow \mathrm{S}_{n}+\mathrm{S}_{0}$,

$\mathrm{S}_{n} \rightarrow \mathrm{S}_{0}+h \nu_{\mathrm{DF} n}$.

Process (1) is called triplet-triplet annihilation (TTA) [3] or triplet fusion [4]. The delayed fluorescence due to TTA is called delayed fluorescence of the pyrene type ( $P$ type) in order to distinguish it from the delayed fluorescence of the eosin type (E

\footnotetext{
1 To whom correspondence should be addressed.

2 Present address: Max-Planck-Institut für Chemie, Abteilung Luftchemie, Saarstrasse 23, D-6500 Mainz, FRG.
}

type) due to thermally activated intersystem crossing $T_{1} \leadsto S_{1}[1]$. Since in this paper only the $P$ type delayed fluorescence is of interest, we omit the specification "P type" in the following.

With many compounds the primary excited state populated by TTA is an upper excited singlet state $S_{n}$ $(n>1)$, and a delayed $S_{n} \rightarrow S_{0}$ fluorescence can be observed [2]. However, due to the very fast internal conversion $S_{n} \rightarrow S_{1}$, in general only the delayed $S_{1} \rightarrow S_{0}$ fluorescence is easy to detect. The delayed $S_{n} \rightarrow S_{0}$ fluorescence $(n>1)$ is typically by a factor $\leqslant 10^{-4}$ weaker than the delayed $S_{1} \rightarrow S_{0}$ fluorescence of a strongly fluorescent compound [2]. We illustrate this point and the main objective of this paper in fig. 1 by the complete spectrum of the delayed luminescence from pyrene in methylcyclohexane at $193 \mathrm{~K}$. From left to right, the delayed-luminescence spectrum consists of the following components:

(a) $\tilde{\nu} \$ 17200 \mathrm{~cm}^{-1}$ : structured phosphorescence with a background of delayed excimer fluorescence;

(b) $17200 \mathrm{~cm}^{-1} \leqslant \tilde{\nu} \leqslant 23000 \mathrm{~cm}^{-1}$ : dominant 


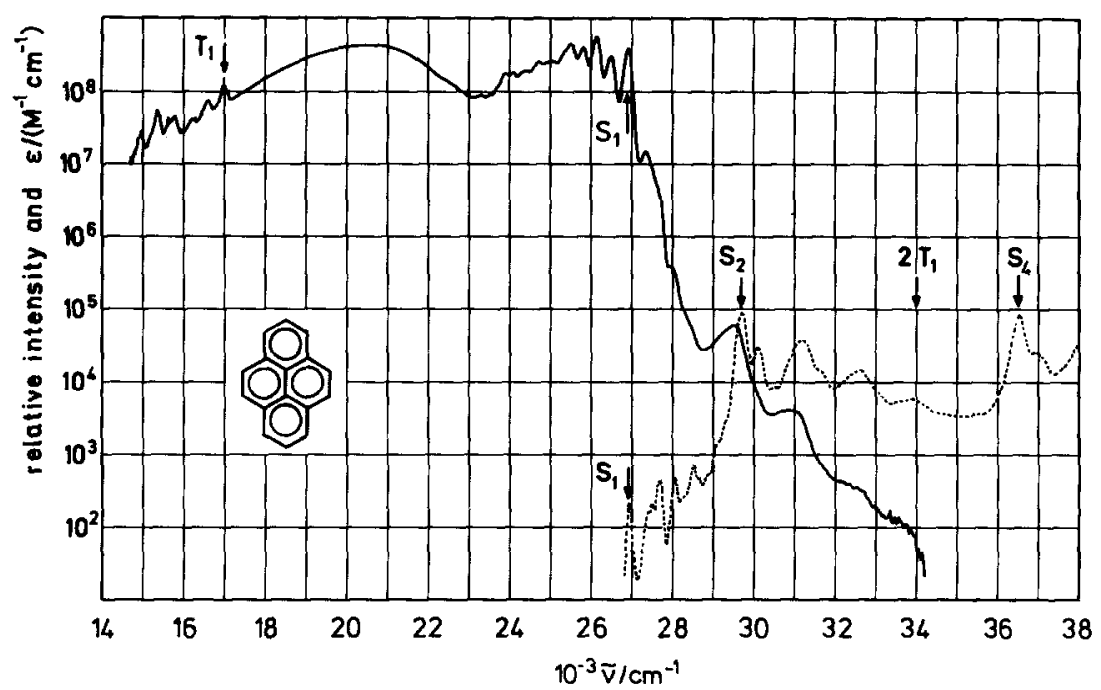

Fig. 1. Spectrally corrected spectrum of the delayed luminescence $\left(-\right.$ ) from a solution of $1.0 \times 10^{-5} \mathrm{M}$ pyrene in methylcyclohexane at $193 \mathrm{~K}$ and absorption spectrum $\epsilon(\tilde{\nu})(-)$ of pyrene at the same temperature. Apart from changes in the annotation, the figurc is identical with the upper part of fig. 4 in ref. [2].

structureless delayed excimer fluorescence;

(c) $23000 \mathrm{~cm}^{-1} \leqslant \tilde{\nu} \$ 27100 \mathrm{~cm}^{-1}:$ structured delayed $S_{1} \rightarrow S_{0}$ monomer fluorescence;

(d) $27100 \mathrm{~cm}^{-1} \leqslant \tilde{\nu} \leqslant 28300 \mathrm{~cm}^{-1}$ : hot bands of delayed $\mathrm{S}_{1} \rightarrow \mathrm{S}_{0}$ fluorescence;

(e) $28300 \mathrm{~cm}^{-1} \leqslant \tilde{\nu} \leqslant 29800 \mathrm{~cm}^{-1}$ : delayed monomer-like $S_{2} \rightarrow S_{0}$ fluorescence;

(f) $29800 \mathrm{~cm}^{-1} \leqslant \tilde{\nu} \leqslant 32000 \mathrm{~cm}^{-1}$ : dominant nonequilibrium hot bands of delayed $S_{2} \rightarrow S_{0}$ fluorescence;

(g) $32000 \mathrm{~cm}^{-1} \lesssim \tilde{\nu} \leqslant 34000 \mathrm{~cm}^{-1}$ : an extremely weak delayed luminescence near $2 E\left(\mathrm{~T}_{1}\right)$.

The assignment of the spectral ranges $(d)-(f)$ is based on the comparison of the delayed-luminescence spectrum with the absorption spectrum of pyrene in the same spectral ranges. The assignment of the high-energy range $(\mathrm{g})$ of the delayed luminescence near $2 E\left(T_{1,0}\right)$ is less straightforward. In this paper we investigate to what extent the high-energy regions of the delayed luminescence from pyrene and other aromatic compounds can be assigned to direct radiative triplet-triplet annihilation.

Knox and Swenberg [5] have shown that apart from the nonradiative TTA (1) a direct radiative TTA is conceivable:

$\mathrm{T}_{\mathrm{I}}+\mathrm{T}_{\mathrm{I}} \rightarrow \mathrm{S}_{\mathrm{0}}+\mathrm{S}_{\mathrm{0}}+h \nu_{\mathrm{rad}}$.
Two molecules in $T_{1}$ perform a simultaneous transition to their electronic ground states and emit their electronic excitation energy as a single photon. The quantitative estimates in ref. [5] refer to triplet excitons in anthracene crystals. For the ratio of the rate constants $k_{\mathrm{r}}$ of direct radiative TTA (3) and $k_{\mathrm{nr}}$ of nonradiative TTA (1) in a contact pair of triplets, Knox and Swenberg obtained $k_{\mathrm{r}} / k_{\mathrm{nr}} \approx 10^{-5}$. Jortner and Rice [6,7] calculated for the same system $k_{\mathrm{r}}$ / $k_{\mathrm{nr}} \approx 10^{-7}$. Mavroyannis [8] confirmed the qualitative conclusions of refs. [5-7] on the basis of a more general theoretical investigation including also the inverse of radiative TTA (the direct simultaneous creation of two triplet excitons by the absorption of one photon). He made, however, no quantitative predictions for real systems.

The term direct radiative TTA was chosen [5], because sometimes (e.g. in ref. [9]) the combination of processes ( 1 ) and (2) was called "radiative TTA" in contrast to the "nonradiative" triplet channel of TTA (see below). We omit the specification "direct" in the following.

There is no doubt that cooperative two-molecule one-photon processes similar to (3) do occur in nature. The best known examples are the simultaneous excitation of two oxygen molecules by the absorption 
of one photon [10] and its counterpart, the dimol luminescence of metastable singlet oxygen, $2 \mathrm{O}_{2}\left({ }^{1} \Delta_{\mathrm{g}}\right) \rightarrow 2 \mathrm{O}_{2}\left({ }^{3} \Sigma_{\mathrm{g}}^{-}\right)+1 h \nu_{\mathrm{rad}}$ [11]. The observability of this luminescence results mainly from the absence of a fast competing bimolecular annihilation process of singlet oxygen. With aromatic compounds, the conditions for the observation of the analogous radiative TTA (3) are much less favourable. Even if because of the energy relation $2 E\left(\mathrm{~T}_{1}\right)<E\left(\mathrm{~S}_{1}\right)$ the singlet channel $(1)$ of nonradiative TTA is energy forbidden, still the triplet channel of nonradiative TTA, $T_{1}+T_{1} \rightsquigarrow T_{j}+S_{0}(j>1)$, may be energy allowed and preclude a high yield of radiative TTA.

Pentacene is one of the few aromatic compounds for which the relation $2 E\left(\mathrm{~T}_{1}\right)<E\left(\mathrm{~S}_{1}\right)$ holds with certainty. For pentacene Swenberg [12] estimated $k_{\mathrm{r}} \approx 50 \mathrm{~s}^{-1}$, and he concluded from this very low value that radiative TTA in pentacene crystals cannot be observed (for comparison: for anthracene Knox and Swenberg [5] obtained $k_{\mathrm{r}} \approx 5 \times 10^{4} \mathrm{~s}^{-1}$ ). In agreement with this estimate Schott and Delannoy [13] did not find any indication of radiative TTA in a pentacene film; they estimated the contribution of radiative TTA to the total TTA at a fraction of less than $10^{-6}$. A promising compound with respect to the detection of radiative TTA was azulene [14], for which the energy relation $2 E\left(\mathrm{~T}_{1}\right)<E\left(\mathrm{~S}_{2}\right)$ holds. However, instead of the expected radiative TTA one observes delayed monomer-like $S_{2} \rightarrow S_{0}$ fluorescence resulting from thermally activated TTA $T_{1}+T_{1} \rightarrow S_{2}+S_{0}$ [15].

In the normal case of an aromatic compound represented by pyrene, $2 E\left(T_{1}\right)$ considerably exceeds $E\left(\mathrm{~S}_{1}\right)$, and the spectrum of the delayed luminescence due to TTA extends up to $2 E\left(\mathrm{~T}_{1}\right)$ [2]. The main objectives of this paper have been (1) to propose criteria for the assignment of the extremely weak delayed luminescence near $2 E\left(\mathrm{~T}_{1}\right)$ to either radiative TTA or delayed fluorescence and (2) to apply these criteria to the delayed luminescence from liquid solutions of pyrene, phenazine, anthracene, 9methylanthracene, and 1,2-benzanthracene. In this connection we discuss the consequences of nonradiative TTA being a special case of Dexter energy transfer.

Apart from these main objectives, the paper is supplementary to ref. [2]. We treat several aspects of the delayed excimer fluorescence from pyrene, and we show that in general the lifetime of an upper excited singlet state $S_{n}$ cannot be calculated from the quantum yield of the delayed $S_{n} \rightarrow S_{0}$ fluorescence - in contrast to the procedure proposed in ref. [2].

\section{Qualitative and quantitative aspects of the spectra of radiative triplet-triplet annihilation and delayed fluorescence}

\subsection{Prediction of the spectrum of radiative triplet- triplet annihilation}

For simplicity we consider only the interaction of triplet molecules in their vibrational ground state $\left(T_{1,0}\right)$, and for the present we neglect the spectral shift resulting from triplet-triplet interaction. The most obvious feature of radiative TTA should be a delayed emission at $2 E\left(\mathrm{~T}_{1,0}\right)$ due to the double 0,0 transition $2 \mathrm{~T}_{1,0} \rightarrow 2 \mathrm{~S}_{0,0}+1 h \nu_{\mathrm{rad}}\left(0^{\prime}, 0^{\prime \prime}\right)$ (the symbol $0^{\prime}, 0^{\prime \prime}$ refers to the final vibrational states of the two molecules). Apart from the double 0,0 transition, vibronic transitions $2 \mathrm{~T}_{1,0} \rightarrow \mathrm{S}_{0, v^{*}}+\mathrm{S}_{0, \nu^{\prime \prime}}+1 h \nu_{\text {rad }}\left(v^{\prime}, v^{\prime \prime}\right)$ must occur. The objective of this section is to derive an expression for the relative intensity of these vibronic transitions.

For cooperative electronic transitions in a pair of centrosymmetric molecules, Jortner and Rice [6] have derived an approximate selection rule, which is based on the assumption of a dominant dipole-dipole term in the intermolecular Coulomb interaction and of negligible exchange interaction. According to this selection rule, in a cooperative transition, of the two individual transitions performed in the two interacting molecules one is parity-forbidden and the other one parity-allowed. If both individual transitions are spin-allowed and if the parity-allowed transition is strongly dipole-allowed, this selection rule is likely to be a good approximation. Applied to radiative TTA, this selection rule would mean that no double 0,0 transition is observable. However, since in radiative TTA both individual transitions are spinforbidden, exchange interaction and higher multipole components in the Coulomb interaction are likely to be the dominant interactions. Therefore, we make the basic assumption that radiative TTA be not subjected to a parity selection rule. By assuming sym- 
metry selection to be predominantly intramolecular, the relative intensities of vibronic transitions of radiative TTA can be easily calculated from the phosphorescence spectrum, if two assumptions (A) are made, which both imply the validity of the Born-Oppenheimer approximation:

(A1) In radiative TTA, the final vibrational states $\mathrm{S}_{0, v^{\prime}}$ and $\mathrm{S}_{0, v^{\prime \prime}}$ of two interacting molecules in $\mathrm{T}_{1,0}$ are uncorrelated in the sense that the relative probability $p\left(v^{\prime}, v^{\prime \prime}\right)$ of a particular combination of final states is given by

$p\left(v^{\prime}, v^{\prime \prime}\right) \propto f_{\mathrm{FC}}^{\prime}\left(0^{\prime}, v^{\prime}\right) f_{\mathrm{FC}}^{\prime \prime}\left(0^{\prime \prime}, v^{\prime \prime}\right) \tilde{\nu}_{\mathrm{rad}}^{3}\left(v^{\prime}, v^{\prime \prime}\right)$.

Here $f_{\mathrm{FC}}^{\prime}$ and $f_{\mathrm{FC}}^{\prime \prime}$ are Franck-Condon factors that are independent of each other, and $\tilde{\nu}_{\text {rad }}\left(v^{\prime}, v^{\prime \prime}\right)$ is given by

$h c \tilde{\nu}_{\mathrm{rad}}\left(v^{\prime}, v^{\prime \prime}\right)=2 E\left(\mathrm{~T}_{1,0}\right)-E_{v^{\prime}}-E_{v^{\prime \prime}}$,

where $h$ is Planck's constant, $c$ is the speed of light, and $E_{v^{\prime}}$ and $E_{v^{\prime \prime}}$ are the ground-state vibrational energies. For the factor $\tilde{\nu}_{\text {rad }}^{3}$ in eq. (4) cf. e.g. ref. [16].

(A2) The vibrations active in radiative TTA and the respective Franck-Condon factors are the same as those in the normal $T_{1} \rightarrow S_{0}$ phosphorescence spectrum. That means, apart from the effect of the $\tilde{\nu}^{3}$ factor in spontaneous radiative transitions [16], the transition of the individual molecule from $T_{1,0}$ to a final state $\mathrm{S}_{0, v}$ has the same relative probability as in the phosphorescence.

A consequence of assumptions (A1) and (A2) is that a photon of radiative TTA ( $\left.\tilde{\nu}_{\text {rad }}\right)$ can be considered as being composed of two phosphorescence photons $\left(\tilde{\nu}_{\mathrm{P}}\right)$,

$\tilde{\nu}_{\mathrm{rad}}=\tilde{\nu}_{\mathrm{P}}^{\prime}+\tilde{\nu}_{\mathrm{P}}^{\prime \prime}$.

As a first approximation to the spectrum of radiative TTA, a spectrum $I_{\text {rad }}^{*}(\tilde{\nu})$ is obtained by summation over all combinations of $\tilde{\nu}_{\mathrm{P}}^{\prime}$ and $\tilde{\nu}_{\mathrm{P}}^{\prime \prime}$ that satisfy condition (6). For a continuous phosphorescence spectrum the summation is replaced by an integration,

$I_{\text {rad }}^{*}(\tilde{\nu})=C \tilde{\nu}^{3} \int_{\nu_{\mathrm{a}}}^{\nu_{\mathrm{b}}} F_{\mathrm{P}}\left(\tilde{\nu}^{\prime}\right) F_{\mathrm{P}}\left(\tilde{\nu}-\tilde{\nu}^{\prime}\right) \mathrm{d} \tilde{\nu}^{\prime}$,

where $C$ is a constant factor and $F_{\mathbf{P}}\left(\tilde{\nu}^{\prime}\right)$ is related to the phosphorescence spectrum $I_{\mathrm{P}}\left(\tilde{\nu}^{\prime}\right)$ by

$F_{\mathrm{P}}\left(\tilde{\nu}^{\prime}\right)=\left(\tilde{\nu}^{\prime}\right)^{-3} I_{\mathrm{P}}\left(\tilde{\nu}^{\prime}\right)$.
Eq. (7) means that the spectrum of radiative TTA should be similar to the convolution of the phosphorescence spectrum with itself. If the phosphorescence spectrum is known for $\tilde{\nu}^{\prime} \geqslant \tilde{\nu}_{\mathrm{a}}$ and is equal to zero for $\tilde{\nu}^{\prime} \geqslant \tilde{\nu}_{\mathrm{b}}$, then $I_{\text {rad }}^{*}(\tilde{\nu})$ can be calculated in the spectral range

$\tilde{\nu}_{\mathrm{a}}+\tilde{\nu}_{\mathrm{b}} \leqslant \tilde{\nu} \leqslant 2 \tilde{\nu}_{\mathrm{b}}$

Finally, the true spectrum $I_{\text {rad }}(\tilde{\nu})$ of radiative TTA must still differ from $I_{\text {rad }}^{*}(\tilde{\nu})$ at least in two respects. Firstly, the triplet-triplet interaction must lead to a red shift of $I_{\text {rad }}(\tilde{\nu})$ relative to $I_{\text {rad }}^{*}(\tilde{\nu})[3,5]$. Secondly, the nonuniformity of triplet-triplet interaction (due to a variation of distance and relative orientation) in radiative TTA must lead to spectral broadening of $I_{\mathrm{rad}}(\tilde{\nu})$ relative to $I_{\mathrm{rad}}^{*}(\tilde{\nu})$. In order to account for both effects, we make a third assumption:

(A3) The best approximation $I_{\text {rad }}(\tilde{\nu})$ to the spectrum of radiative TTA is obtained by a red shift of $I_{\mathrm{rad}}^{*}(\tilde{\nu})$ by $\Delta \tilde{\nu}_{\mathrm{TT}}$,

$I_{\mathrm{rad}}^{\dagger}(\tilde{\nu})=I_{\mathrm{rad}}^{*}\left(\tilde{\nu}+\Delta \tilde{\nu}_{\mathrm{TT}}\right)$,

and by convolution of $I_{\text {rad }}^{\ddagger}(\tilde{\nu})$ with a normalized Gaussian accounting for additional spectral broadening:

$$
\begin{aligned}
& I_{\mathrm{rad}}(\tilde{\nu})=\frac{1}{\sqrt{2 \pi \sigma^{2}}} \int I_{\mathrm{rad}}^{\ddagger}\left(\tilde{\nu}^{\prime}\right) \\
& \quad \times \exp \left[-\left(\tilde{\nu}^{\prime}-\tilde{\nu}\right)^{2} / 2 \sigma^{2}\right] \mathrm{d} \tilde{\nu}^{\prime} .
\end{aligned}
$$

$\Delta \tilde{\nu}_{\mathrm{TT}}$ and the Gaussian half-width $B_{\mathrm{G}}=2 \sqrt{2 \ln 2} \sigma$ are the only adjustable parameters in the calculation of the spectrum of radiative TTA.

This simple model for the prediction of the spectrum of radiative TTA is certainly restricted to large molecules, where intramolecular motion may be considered as independent of intermolecular motion, which is an implication of assumption (A2). It is expedient first to apply the model to several compounds and then to discuss its limitations (cf. section 5.6).

\subsection{Peculiarities of delayed-fluorescence spectrum between $E\left(S_{1,0}\right)$ and $2 E\left(T_{1,0}\right)$}

Let $\left\{\mathbf{S}_{i, v}\right\}$ be the set of vibronic states accessible to TTA, either directly or by subsequent relaxation processes. Then the spectrum $I_{\mathrm{DF}}(\tilde{\nu})$ of the delayed flu- 
orescence due to TTA can be written in the form

$I_{\mathrm{DF}}(\tilde{\nu})=C \sum_{i, v} F_{i, v}(\tilde{\nu}) \bar{p}_{i, v} / \tau_{i, v}^{\mathrm{r}}$,

where $C$ is a constant, $F_{i, v}(\tilde{\nu})$ is the normalized spectrum of the fluorescence from the state $S_{i, n} \bar{p}_{i, v}$ is the relative steady-state probability for the population of $S_{i, v}$ and $\tau_{i, v}^{\mathrm{r}}$ is the radiative lifetime of $S_{i, v}$ The actual application of eq. (12) to the calculation of $I_{\mathrm{DF}}(\tilde{\nu})$ above $E\left(\mathrm{~S}_{1,0}\right)$ is not possible on the following grounds:

(1) When several excited singlet states $S_{i}$ are energetically accessible by TTA, it is not possible to predict with certainty the probability $p_{i}$ of the primary population of $S_{i}$ by TTA $[2,3,12,17]$. For example, in the case of pyrene $\left(2 \mathrm{~T}_{1,0}: 34000 \mathrm{~cm}^{-1}\right)$, apart from the state $S_{2}\left(B_{3 u}\right)\left(29700 \mathrm{~cm}^{-1}\right)$, the state $S_{3}\left(B_{1 \mathrm{~g}}\right)$ $\left(33200 \mathrm{~cm}^{-1}[18]\right)$ can be populated by TTA.

(2) If the state $S_{i}$ is created by TTA, the energy difference $2 E\left(\mathrm{~T}_{1,0}\right)-E\left(\mathrm{~S}_{i, 0}\right)$ is distributed as vibrational energy [3] over the two interacting molecules, $\mathrm{T}_{1,0}+\mathrm{T}_{1,0} \rightarrow \mathrm{S}_{i, \nu}+\mathrm{S}_{0, w}$. The relative probabilities $p_{i, v}$ of the creation of vibronic states $S_{i, v}$ by TTA are not known. Moreover, if $S_{i}$ is an upper excited state $(i>1)$ and if the energy difference between $S_{i}$ and $S_{i-1}$ is of the order of a few $1000 \mathrm{~cm}^{-1}$, then due to strong intermediate vibronic coupling [19-21] neither the vibronic states $S_{i, \nu}$ nor their radiative lifetimes $\tau_{i, v}^{\mathrm{r}}$ are well known (see also section 5.7). Pyrene is an example for strong intermediate vibronic $\mathrm{S}_{2}-\mathrm{S}_{1}$ coupling [22,23].

(3) In order to calculate from the primary probabilities $p_{i, v}$ of vibronic states $S_{i, v}$ the steady-state probabilities $\bar{p}_{i, v}$, one would need the relaxation pathways and rate constants of primary and secondary vibronic states, which are not known.

(4) Finally, an estimate of $F_{i, \nu}(\tilde{\nu})$ would be also uncertain, because the delayed fluorescence is in principle always the fluorescence from pairs of interacting molecules. Since during the short lifetime of vibronic states $\mathbf{S}_{i, v}$ the two interacting molecules cannot move far apart, the interaction can be considerable and has to be taken into account.

In conclusion, practically all data that are required for the calculation of the spectrum of the delayed fluorescence above $E\left(\mathrm{~S}_{1,0}\right)$ are not available. Nevertheless qualitative statements on the spectrum of the delayed fluorescence in the high-energy region close to
$2 E\left(T_{1,0}\right)$ can be made. By neglecting part of the intermolecular correlations, we can treat nonradiative TTA as a special case of Dexter energy transfer [24]. This approximation will be the better the smaller the non-Coulombic interactions between the annihilating molecules are, i.e. the larger the distance between them is. If this approach is chosen, then in principle an inhomogeneously broadened population spectrum of vibronic states $S_{i, v}$ can be calculated from the overlap of the $T_{1} \rightarrow S_{0}$ phosphorescence spectrum with the $T_{1} \rightarrow S_{i}$ absorption spectrum. Though the latter spectrum is in general not known, nevertheless by this approach important qualitative conclusions on the spectrum of the delayed fluorescence close to $2 E\left(T_{1,0}\right)$ can be drawn, which depend on the symmetry of the molecules.

Case A: Molecules with no center of symmetry

(I) As long as no contrary selection rule is known, one may assume that by nonradiative TTA in part the same vibronic states $S_{n, \nu}$ are populated as by onephoton $S_{0} \rightarrow S_{n}$ absorption. Let $\left\{S_{n, \nu_{0}}\right\}$ be a set of such states satisfying the relation $E\left(S_{n, v o}\right) \approx 2 E\left(T_{1,0}\right)$ (for simplicity we here omit the red shift $\Delta \tilde{\nu}_{\mathrm{TT}}$ resulting from triplet-triplet interaction ):

$\left\{\mathrm{T}_{1,0}+\mathrm{T}_{1,0} \rightarrow \mathrm{S}_{n, v 0}+\mathrm{S}_{0,0}\right\}$.

Then in principle at the energy $2 E\left(\mathrm{~T}_{1,0}\right)$ a delayed $\left\{\mathbf{S}_{n, v 0} \rightarrow \mathbf{S}_{0,0}\right\}$ fluorescence must be observable. That means, with respect to the high-energy bound of the spectrum, delayed fluorescence cannot be distinguished from radiative TTA.

(II) If nonradiative TTA is a special case of Dexter energy transfer, then the phosphorescence spectrum imposes its structure on the distribution function of the probabilities $p_{n, \nu}$ of the primary population of vibronic states $S_{n, v}$ by TTA. Hence the second set of vibronic states $S_{n, \nu 1}$ results from the TTA

$\left\{T_{1,0}+T_{1,0} \rightsquigarrow S_{n, v_{1}}+S_{0, w_{1}}\right\}$,

where $w_{1}$ stands for the lowest vibration active in phosphorescence. Process (14) leads to the second delayed-fluorescence band $\left\{\mathbf{S}_{n, v_{1}} \rightarrow \mathrm{S}_{0,0}\right\}$ located by $\tilde{\nu}_{w 1}$ below the first one. Note that the set of vibronic transitions $\left\{S_{n, \nu_{0}} \rightarrow S_{0, w_{1}}\right\}$ also contributes to the intensity of the second band. Thus at least close to $2 E\left(\mathrm{~T}_{1,0}\right)$ the vibrational structures of the spectra of delayed fluorescence and of radiative TTA would be identical. 
As a consequence of (II), the spectrum of the delayed fluorescence may have a structure near $2 E\left(\mathrm{~T}_{1,0}\right)$ even if the normal absorption spectrum has no structure in this spectral range. A pronounced structure in the phosphorescence spectrum is a sufficient condition for a structure in the delayed-fluorescence spectrum, as long as the absence of structure in the absorption spectrum is due to vibrational congestion and inhomogeneous broadening - and not to extremely strong homogeneous broadening.

In conclusion, in case A neither the extension of the spectrum of a delayed luminescence up to $2 E\left(T_{1,0}\right)$ nor its structure close to this energy can be used as criteria for the distinction between radiative TTA and delayed fluorescence. The similarity between the spectrum of the observed delayed luminescence and the calculated spectrum of radiative TTA is left as the only criterion for the distinction between both kinds of delayed luminescence. An example for case $\mathrm{A}$ is 1,2-benzanthracene (cf. section 5.5).

Case B: Molecules with a center of symmetry

(1) Let $T_{1,0}$ and $S_{n, 0}$ be u states observable in the 0,0 transitions of the $T_{1} \rightarrow S_{0}$ phosphorescence and of one-photon $S_{0} \rightarrow S_{n}$ absorption. Then the transition $\mathrm{T}_{1,0}^{(u)} \rightarrow \mathbf{S}_{n, v_{\mathrm{g}}}^{(u)}$ is parity-forbidden $\left(v_{\mathrm{g}}\right.$ or $v_{\mathrm{u}}$ stands for a combination of vibrations with total $\mathrm{g}$ or $\mathrm{u}$ symmetry). If we again consider nonradiative TTA as a special case of Dexter energy transfer, then vibronic states $S_{n, \nu_{\mathrm{g}}}^{(u)}$ observable in the $\mathbf{S}_{\mathbf{d}, 0}^{(\mathrm{g})} \rightarrow \mathbf{S}_{n, \nu_{\mathrm{z}}}^{(\mathrm{u})}$ absorption spectrum are not expected to be created by nonradiative TTA. On the other hand, vibronic states $S_{n, v_{u}}^{(g)}$ with total $\mathrm{g}$ symmetry are expected to be populated by nonradiative TTA, but the fluorescence $S_{n, v_{u}}^{(\mathrm{g})} \rightarrow S_{\delta, 0}^{(\mathrm{g})}$ is parity-forbidden (in order to make the fluorescence transition allowed, one quantum of a u vibration must remain in the molecule).

(2) Let $S_{m, 0}^{(g)}$ be a $g$ state, which is not observed in the one-photon absorption spectrum. Then the transition $T_{l, 0}^{(\mathrm{u})} \rightarrow S_{m, v_{\mathrm{g}}}^{(\mathrm{g})}$ is parity-allowed, but the fluorescence $S_{m, \nu_{z}}^{(g)} \rightarrow S_{\delta, 0}^{(g)}$ is parity-forbidden; again one quantum of a vibration must remain in the molecule in order to make the fluorescence transition allowed.

From items (1) and (2) follows the conclusion, that the nonradiative annihilation of two $T(u), 0$ states cannot lead to an observable delayed fluorescence at the energy $2 E\left(\mathrm{~T}_{1,0}\right)$, if the treatment of nonradiative TTA as Dexter energy transfer is justified. Therefore, within this approximation and in the case of centrosymmetric molecules, the observation of a delayed luminescence at about $2 E\left(\mathrm{~T}_{1,0}\right)$ at low temperatures is a strong argument for the assignment of this delayed luminescence to radiative TTA. Examples for this case are pyrene, phenazine and anthracene (cf. sections 5.1-5.3).

(3) Let $T(g)$, be a g state and $S_{n, v_{\mathrm{g}}}^{(\mathrm{g})}$ a vibronic state with total $\mathrm{u}$ symmetry. Then the 0,0 transition $T_{l, 0}^{(g)} \rightarrow S_{0,0}^{(g)}$ of the phosphorescence is parity-forbidden. An example for this case is biphenylene [25]. Let $T^{(g)} \rightarrow S_{0, v_{u}}^{(u)}$ be the false origin of the phosphorescence spectrum. The transitions $T(\xi) \rightarrow S_{n, \nu_{g}}^{(u)}$ and $\mathbf{S}_{n, v_{\mathbf{z}}}^{(\mathrm{u})} \rightarrow \mathbf{S}_{d, 0}^{(\mathrm{g})}$ are parity-allowed. That means, since in nonradiative TTA the vibrational energy $E\left(v_{\mathrm{u}}\right)$ remains in the ground-state molecule, the high-energy bound of the delayed fluorescence would be at $2 E\left(\mathrm{~T}_{1,0}\right)-E\left(v_{\mathrm{u}}\right)$. Since in this case the high-energy bound of radiative TTA would be at $2 E\left(\mathrm{~T}_{1,0}\right)$ $-2 E\left(v_{\mathrm{u}}\right)$, the observation of a delayed luminescence at $2 E\left(T_{1,0}\right)-E\left(v_{\mathrm{u}}\right)$ would be a strong argument for the assignment of this delayed luminescence to delayed fluorescence resulting from nonradiative TTA.

\section{Choice of experimental conditions and compounds}

The main aim of this investigation has been to measure and interpret the high-energy region of the delayed luminescence due to TTA from suitable compounds in the spectral range close to the energy $2 E\left(T_{1,0}\right)$. The interpretation of observed spectra is greatly facilitated, if most of the interacting molecules in $T_{1}$ are in their vibrational ground states $T_{1,0}$ (at least with respect to the vibrations active in nonradiative or radiative TTA and in phosphorescence or fluorescence). For this reason it is desirable to measure the spectrum of the delayed luminescence at the lowest temperature possible. In practice the choice of solvents was limited to compounds with a boiling point still above room temperature. With respect to the alkanes, the $C_{5}$ alkanes are most suitable, and among them isopentane has the lowest melting point $(113 \mathrm{~K})$. We chose a mixture of isopentane and cyclopentane $(7: 3)$ as solvent, because it permitted us to perform experiments still below the melting point of isopentane down to about $105 \mathrm{~K}$.

A low temperature entails a moderate or high vis- 
cosity of the solvent. From a physical point of view, a high viscosity is advantageous on the following grounds. An increase of the solvent viscosity leads to an increase of the average distance $\bar{R}$, at which TTA takes place [26-28]. With respect to radiative TTA that means the spectral shift resulting from triplettriplet interaction and the spectral broadening due to the spread of triplet-triplet interaction energies should become smaller with increasing viscosity. With respect to nonradiative TTA and the delayed fluorescence from short-lived upper excited singlet states, an increase of $\bar{R}$ has the advantage, that the delayed fluorescence becomes more monomer-like and hence more easy to assign. From an experimental point of view, a high viscosity has two disadvantages. Firstly, triplet sensitizers can no longer be used, when the triplet energy transfer from the sensitizer is too slow; that means, compounds like biphenylene [25] with a very low quantum yield of intersystem crossing $S_{1} \leadsto T_{1}$ cannot be investigated. Secondly, for the measurement of the extremely weak delayed luminescence near $2 E\left(T_{1,0}\right)$ a high rate of TTA is required. At high viscosities, the intensity-limiting factor is not the power of the excitation light source but the rate of TTA (for a discussion of this point cf. ref. [25]).

The choice of aromatic compounds has been restricted by the following requirements:

(1) sufficient solubility $\left(\approx 1 \times 10^{-5} \mathrm{M}\right)$ in isopentane/cyclopentane at $110 \mathrm{~K}$;

(2) sufficient quantum yield ( $~(0.2)$ of intersystem crossing $S_{1} \leadsto T_{1}$ at low temperatures;

(3) high molar absorption coefficient at one of the available excitation wavelengths;

(4) high photostability at the chosen excitation wavelength.

Pyrene was chosen for this investigation for two reasons: Its spectrum of the delayed luminescence due to TTA extends up to $2 E\left(\mathrm{~T}_{1,0}\right)[2]$, and it is an example for a compound with a $g$ state accessible by TTA [18].

Phenazine was chosen as an example of a strongly phosphorescent compound. If there is a connection between the rate constants of unimolecular radiative triplet decay (phosphorescence) and radiative TTA, one might expect an unusual result for phenazine.

Anthracene was chosen mainly because of the predictions of the relative efficiency of radiative TTA
[5-7]. Except for that, anthracene has been of interest, because only $S_{1}$ is likely to be populated by nonradiative TTA; hence there are no complications in the interpretation of the delayed fluorescence resulting from strong intermediate $S_{n}-S_{n-1}$ coupling (see sections 2.2 and 5.7). 9-methylanthracene was included in this investigation in order to study the effect of a small perturbation of the symmetry of anthracene by a substituent.

1,2-benzanthracene has been of interest in two respects: Firstly, $2 E\left(T_{1,0}\right)$ coincides with the 0,0 transition of a moderately strong absorption band and is only $\approx 1000 \mathrm{~cm}^{-1}$ below the 0,0 transition of a strong absorption band [2]. Secondly, 1,2-benzanthracene has a low symmetry $\left(C_{s}\right)$.

\section{Experimental}

The basic experimental technique has been described in ref. [2]. Here we mention only those points which are important in the present kind of investigation.

Purity of substances. Impurities (Q) of an aromatic compound (A) can be classified according to the energies $E\left({ }^{1} \mathrm{Q}^{*}\right)$ and $E\left({ }^{1} \mathrm{~A}^{*}\right)$ of the lowest excited singlet states and $E\left({ }^{3} \mathrm{Q}^{*}\right)$ and $E\left({ }^{3} \mathrm{~A}^{*}\right)$ of the lowest excited triplet states of $Q$ and $A$ [2]. Here we draw the attention to case (d) of this classification (cf. ref. [2]): $E\left({ }^{1} \mathrm{Q}^{*}\right)>E\left({ }^{1} \mathrm{~A}^{*}\right)$ and $E\left({ }^{3} \mathrm{Q}^{*}\right)>E\left({ }^{3} \mathrm{~A}^{*}\right)$. In a fluid and moderately concentrated solution of $A\left([A] \gtrsim 10^{-4} M\right)$, excitation of $Q$ does not cause trouble in the measurement of the delayed luminescence from $A$ because of the fast and practically irreversible triplet energy transfer from $Q$ to $A$. If the solution is dilute ( $[A] \approx 10^{-5} \mathrm{M}$ ) and moderately viscous $(10 \mathrm{cP} \lesssim$ viscosity $\lesssim 100 \mathrm{cP})$, then the triplet energy transfer from $Q$ to $A$ is no longer fast, and in particular hetero-TTA of ${ }^{3} \mathrm{~A}^{*}$ and ${ }^{3} \mathrm{Q}^{*}$ may give rise to disturbing delayed luminescence in the spectral region close to $2 E\left({ }^{3} \mathrm{~A}^{*}\right)$ (for systematic investigations or applications of hetero-TTA $\mathrm{cf}$. refs. $[14,29,30])$. Whether an impurity of type (d) is disturbing or not, depends on the excitation wavelength. An example is the excitation of anthracene with carbazole as an impurity. The 0,0 transition to $S_{1}$ of carbazole is at $337 \mathrm{~nm}$; hence for excitation of anthracene with all three UV lines of an argon ion 
laser (364, 351 and $333 \mathrm{~nm})$, carbazole would be a disturbing impurity of type (d).

All aromatic compounds were purified by column chromatography, sublimation and zone refining, and in part by other procedures (pyrene [2], phenazine [31 ], anthracene (carbazole-free) and 9-methylanthracene [30], 1,2-benzanthracene [2] ). Isopentane and cyclopentane were rectified over a column ( $1 \mathrm{~m}$ ) and chromatographed with basic aluminium oxide immediately before use.

Samples. Special fluorescence flow cells with a closed circuit $[2,32]$ were used. In these cells a continuous circulation of a solution is maintained by asymmetric cooling (here with cold nitrogen gas). The cross section of a fluorescence flow cell is 3 $\mathrm{mm} \times 10 \mathrm{~mm}$. The path length of the excitation light in the solution is $10 \mathrm{~mm}$, and the average path length of luminescence light in the solution is $1.5 \mathrm{~mm}$; this length is important for estimating luminescence reabsorption. Samples were prepared as described in ref. [33].

Excitation. Samples were excited either with all three groups of lines in the near UV $(364,351$ and $333 \mathrm{~nm}$ ) of an argon ion laser (Spectra Physics, model 171-19) or only with one of them. Typically the total UV power was $\approx 1 \mathrm{~W}$, and the power of the chopped laser beam incident on the sample was of the order of $100 \mathrm{~mW}$ for single-line excitation and of the order of $300 \mathrm{~mW}$ for excitation with all UV lines. The laser beam diameter in the sample was $\approx 1 \mathrm{~mm}$. For the measurement of strong delayed $S_{1} \rightarrow S_{0}$ fluorescences, lower excitation powers were used.

Mirror optics. Because of the large spectral ranges of the delayed luminescences, achromatic optics were essential for the detection of delayed luminescence. In the spectral range of interest, aluminium-coated mirrors without protective layer are best suited for this purpose. For a practical realization cf. ref. [21 ].

Chopper. For the discrimination between prompt and delayed luminescences a mechanical chopper was used with equal intervals for excitation and detection of a delayed luminescence (maximal chopping frequency $10 \mathrm{kHz}$; for details cf. ref. [25]).

Monochromator. When the delayed luminescence near $2 E\left(T_{1}\right)$ is by a factor of $10^{-7}$ weaker than the delayed fluorescence from $S_{1}$ (as in the case of pyrene), the use of a good double monochromator is es- sential. Two home-made double monochromators with holographic gratings were used.

Photomultipliers. Three types of cooled photomultipliers were used: EMI 6256 (blue-sensitive, $\approx 2$ dark counts/s at $-20^{\circ} \mathrm{C}$ ), EMI 9659 QA (S20 photocathode with extended red-sensitivity, sensitive cathode area reduced to $12 \mathrm{~mm}$ diameter with defocusing magnet, $\approx 2$ dark counts $/ \mathrm{s}$ at $-30^{\circ} \mathrm{C}$ ), and Hamamatsu R361-01 (S1 photocathode, infrared-sensitive, $\approx 20$ dark counts $/ \mathrm{s}$ at $-100^{\circ} \mathrm{C}$ ). The intensity of the weakest delayed luminescence bands was $\approx 10$ counts/s. The photon-counting technique was applied, and measured count rates were corrected for dark counts and for pulse overlap at high count rates. A delayed-luminescence spectrum like that of pyrene in fig. 1 is composed of three or more spectra measured with different excitation powers.

Spectral sensitivity correction. The comparison of a calculated spectrum of radiative TTA with an observed spectrum of delayed luminescence is only meaningful, if the experimental spectra of phosphorescence and delayed luminescence are sensitivity corrected. The spectra of phenazine and the phosphorescence spectrum of pyrene refer to the spectralsensitivity calibration described in ref. [34]; all other spectra refer to a spectral-sensitivity calibration similar to that described in ref. [2].

\section{Results and discussion}

\subsection{Delayed luminescence from pyrene}

We measured the spectrum of the delayed luminescence from a $1.0 \times 10^{-5} \mathrm{M}$ solution of pyrene in isopentane/cyclopentane $(7: 3)$ at $110 \mathrm{~K}$. The total spectrum of the delayed luminescence is shown in fig. 2. The phosphorescence spectrum of pyrene in fig. 3 was obtained by the measurement of two spectra of the delayed luminescence, (I) with low and (II) with high excitation intensity, by normalization of both spectra to equal intensity of the delayed fluorescence, and by subtraction of the normalized spectrum (II) from the normalized spectrum (I). The delayed-luminescence spectra in figs. 1 and 2 differ in several respects. We use the same classification of spectral ranges as in section 1 . The most distinctive feature of the spectrum in fig. 2 is the spectral structure of the 


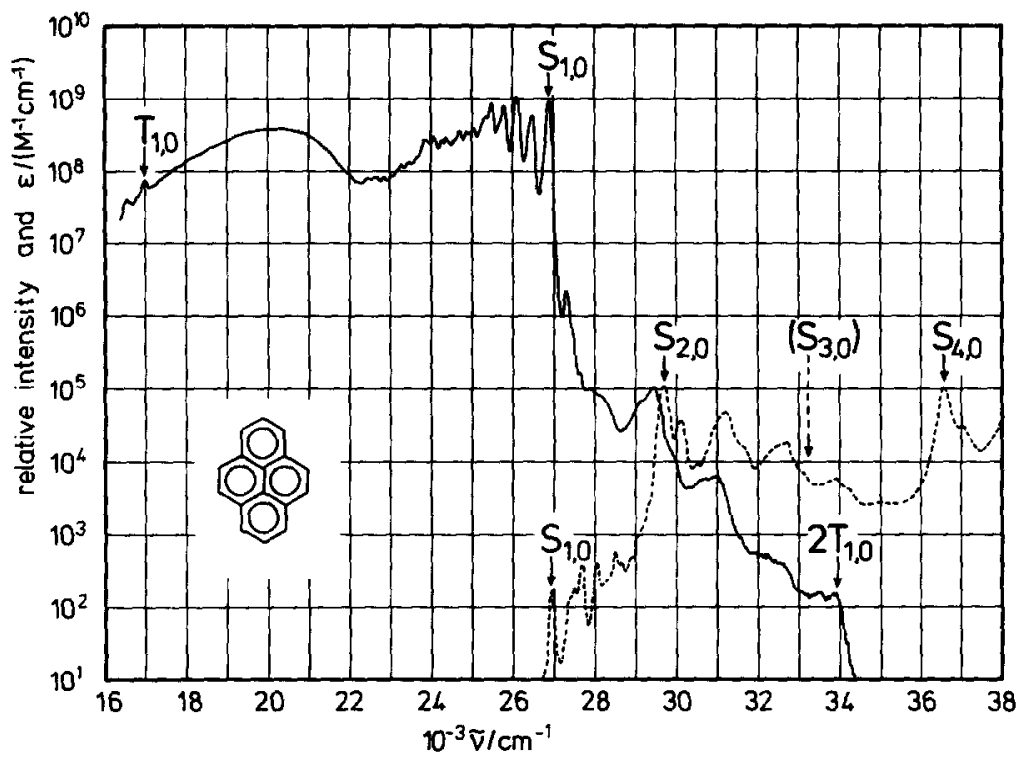

Fig. 2. Spectrally corrected spectrum of the delayed luminescence (-) from a solution of $1.0 \times 10^{-5} \mathrm{M}$ pyrene in isopentane/cyclopentane $(7: 3)$ at $110 \mathrm{~K}$ and absorption spectrum $\epsilon(\tilde{\nu})(-)$ of pyrene at the same temperature (the weak $\mathbf{S}_{0 \rightarrow S_{1}}$ absorption band was measured with pyrene in methylcyclohexane at $193 \mathrm{~K}$ ). Pyrene was excited with the $333 \mathrm{~nm}$ UV lines of an argon ion laser. The measurement of region $(\mathrm{g})$ of the delayed-luminescence spectrum took several days.

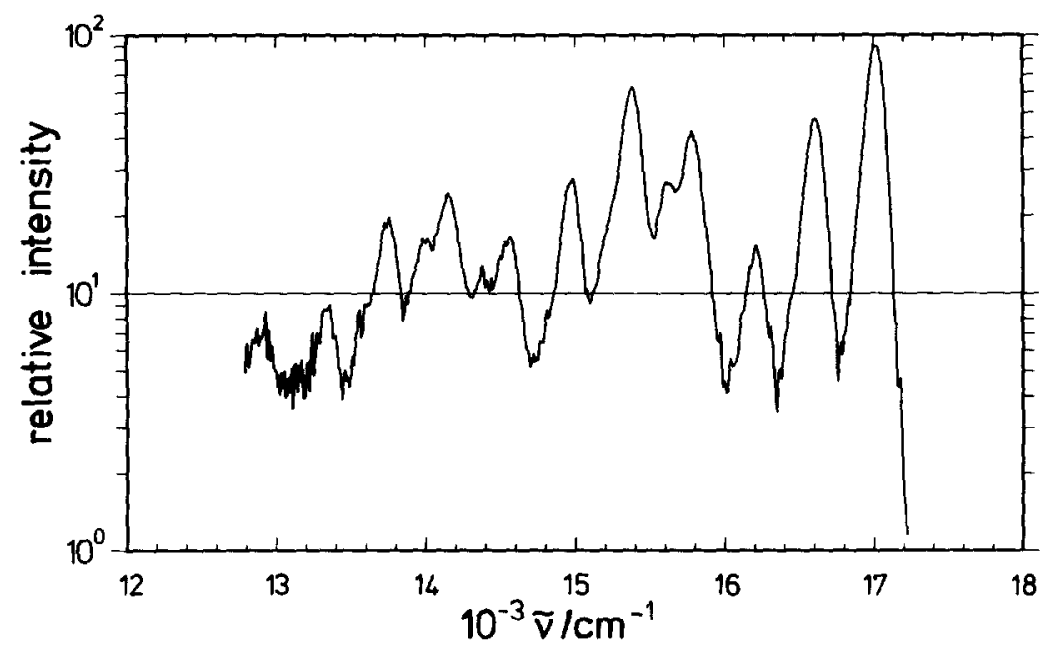

Fig. 3. Spectrally corrected spectrum of the phosphorescence from a solution of $1.0 \times 10^{-5} \mathrm{M}$ pyrene in isopentane/cyclopentane ( $\left.7: 3\right)$ at $110 \mathrm{~K}$.

high-energy energy region $(\mathrm{g})$. We first compare the high-energy region $(\mathrm{g})$ in fig. 2 with the calculated spectrum of radiative TTA and then treat some as- pects of the delayed fluorescence in other spectral ranges. 


\subsubsection{High-energy region of the delayed luminescence from pyrene}

In fig. 4 the regions ( $f$ ) and ( $g$ ) of the delayedluminescence spectrum $I_{\mathrm{DL}}(\tilde{\nu})$ are compared with two calculated spectra $I_{\mathrm{rad}}^{(1)}(\tilde{\nu})$ and $I_{\mathrm{rad}}^{(2)}(\tilde{\nu})$ of radiative TTA. The spectra were calculated from the phosphorescence spectrum in fig. 3 with eq. (11) and with a red shift $\Delta \tilde{\nu}_{T T}=110 \mathrm{~cm}^{-1}$ and the Gaussian widths $B_{G}^{(1)}=0 \mathrm{~cm}^{-1}$ and $B_{G}^{(2)}=166 \mathrm{~cm}^{-1}$. In the spectral range $(\mathrm{g})$ between 32000 and $34000 \mathrm{~cm}^{-1}$ $\left(2 E\left(\mathrm{~T}_{1,0}\right)\right), I_{\mathrm{DL}}(\tilde{\nu})$ and $I_{\text {rad }}^{(2)}(\tilde{\nu})$ have the same structure, and differences between these spectra do not exceed $\pm 30 \%$.

In the case of dominant radiative TTA in range $(\mathrm{g})$, a much better agreement between $I_{\mathrm{DL}}(\tilde{\nu})$ and $I_{\mathrm{rad}}(\tilde{\nu})$ cannot be expected, even if eq. (11) yielded the exact spectrum of radiative TTA. Firstly, there must be some contribution of delayed fluorescence to the total delayed luminescence in range $(\mathrm{g})$. Secondly, uncertainties in the calibration of the spectral sensitivity may cause systematic errors in relative intensities of $I_{\mathrm{DL}}(\tilde{\nu})$ and $I_{\mathrm{rad}}(\tilde{\nu})$ of the order of $\pm 10 \%$ (note that the delayed-luminescence spectrum in fig. 2 and the phosphorescence spectrum in fig. 3 were measured with different spectrometers). Finally, reabsorption of delayed luminescence by pyrene in
$S_{0}$ leads to a systematic error in relative intensities of the order of $\leqslant 5 \%$. Since ground-state depletion was essential, reabsorption of delayed luminescence by molecules in $T_{1}$ has also to be considered; however, in range $(\mathrm{g})$ the molar absorption coefficient of pyrene in $T_{1}$ is smaller than that of pyrene in $S_{0}$ [35].

Apart from the satisfactory agreement between the observed spectrum $I_{\mathrm{DL}}(\tilde{D})$ and the calculated spectrum $I_{\text {rad }}(\tilde{\nu})$ in range $(g)$, the observation of a delayed luminescence at $\approx 2 E\left(\mathrm{~T}_{1,0}\right)$ itself is an additional argument for the assignment of range $(\mathrm{g})$ to dominant radiative TTA. Since pyrene (point symmetry group $D_{2 h}$ ) has a centre of symmetry, according to the symmetry considerations in case $B$ of section 2.2 no delayed fluorescence should be observable at $\approx 2 E\left(\mathrm{~T}_{1,0}\right)$. The red shift $\Delta \tilde{\nu}_{\mathrm{TT}}=110 \mathrm{~cm}^{-1}$ is of the order of magnitude expected by Knox and Swenberg [5]. Therefore, within the frame of the considerations of section 2, all experimental facts are consistent with dominant radiative TTA in the spectral range $(\mathrm{g})$.

Finally, the ratio $k_{\mathrm{r}} / k_{\mathrm{nr}}$ of the rate constants $k_{\mathrm{r}}$ of radiative TTA and $k_{\mathrm{nr}}$ of nonradiative TTA is of interest. We consider only the singlet channel of TTA and assume that direct internal conversion of upper excited singlet states into the electronic ground state

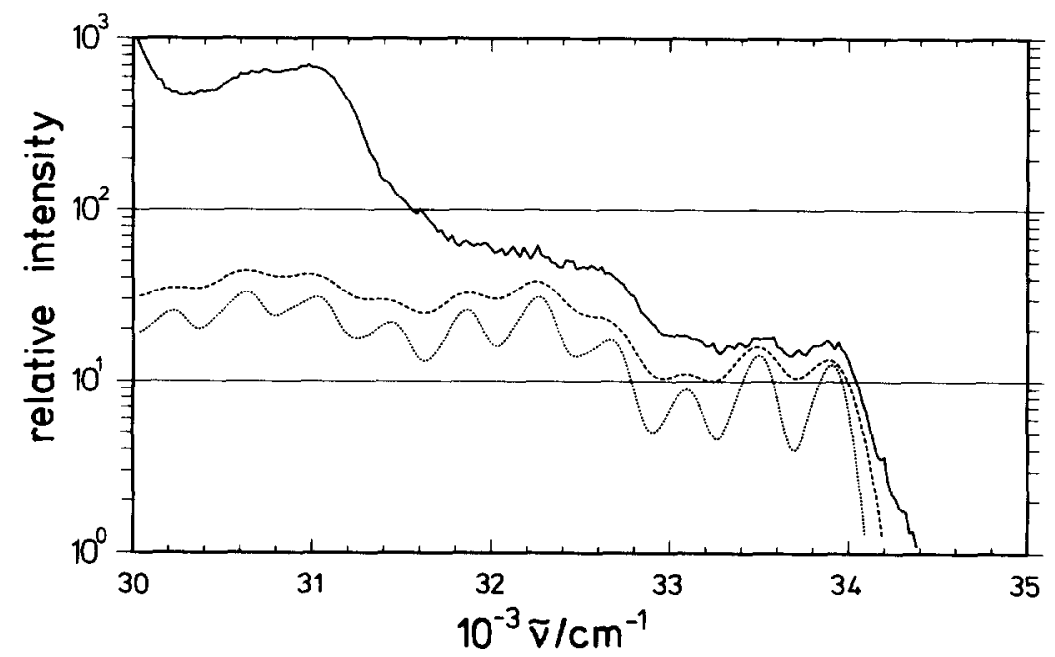

Fig. 4. Comparison of the high-energy region (g) of the delayed-luminescence spectrum of pyrene in fig. 2 (-) with two spectra $I_{\mathrm{rad}}(\tilde{\nu})$ of radiative TTA calculated from the phosphorescence spectrum in fig. 3 with $\Delta \tilde{\nu}_{\mathrm{rT}}=110 \mathrm{~cm}^{-1}$ and different values of $B_{\mathrm{G}}: I_{\mathrm{rad}}^{(1)}(\tilde{\nu})$ with $B g^{(1)}=0 \mathrm{~cm}^{-1}(\ldots)$ and $I_{\mathrm{rad}}^{(2)}(\tilde{\nu})$ with $B_{\mathcal{G}^{\prime}}^{(2)}=166 \mathrm{~cm}^{-1}(--)$. The three spectra are vertically shifted relative to each other. 
can be neglected. Then $k_{\mathrm{r}} / k_{\mathrm{nr}}$ can be written in the form

$k_{\mathrm{r}} / k_{\mathrm{nr}} \approx I_{\mathrm{rad}} /\left(I_{\mathrm{DF} 1} / \phi_{\mathrm{F} 1}+I_{\mathrm{DFE}} / \phi_{\mathrm{FE}}\right)$,

where $I_{\mathrm{rad}}, I_{\mathrm{DF} 1}$ and $I_{\mathrm{DFE}}$ are the spectrally integrated intensities of radiative TTA, delayed monomer fluorescence from $S_{1}$ and delayed excimer fluorescence; $\phi_{\mathrm{F} 1}$ and $\phi_{\mathrm{FE}}$ are the quantum yields of prompt monomer and excimer fluorescence. Since the calculated spectra $I_{\text {rad }}(\tilde{\nu})$ of radiative TTA are in general incomplete, the integration of $I_{\text {rad }}(\tilde{\nu})$ yields $I_{\text {rad }}^{\prime}=$ $p_{\text {rad }} I_{\text {rad }}$ with $p_{\text {rad }}<1$ or

$I_{\mathrm{rad}}=I_{\mathrm{rad}}^{\prime} / p_{\mathrm{rad}}$.

We assume that the delayed luminescence at 33500 $\mathrm{cm}^{-1}$ (figs. 2 and 4 ) can be completely assigned to radiative TTA. For this wavenumber we postulate $I_{\mathrm{DL}}(\tilde{\nu})=I_{\mathrm{rad}}^{(2)}(\tilde{\nu})$. Then, with $p_{\mathrm{rad}} \approx 0.7$ (estimated) and $\phi_{\mathrm{F} 1} \approx \phi_{\mathrm{FE}} \approx 0.7$ (table 7.3 in ref. [36]), eqs. (15) and $(16)$ yield $k_{\mathrm{r}} / k_{\mathrm{nr}} \approx 5 \times 10^{-?}$.

\subsection{2. $S_{2} \rightarrow S_{0}$ and hot-band $S_{1} \rightarrow S_{0}$ delayed fluorescence from pyrene}

The spectrum of the delayed fluorescence at $110 \mathrm{~K}$ (fig. 2) differs from that at $193 \mathrm{~K}$ (fig. 1 ) in several respects. The lower temperature manifests itself most clearly in the lower relative intensity of the hot band $\mathrm{S}_{1, v} \rightarrow \mathrm{S}_{0,0}$ at $27300 \mathrm{~cm}^{-1}$, which corresponds to the lowest totally symmetric vibration of pyrene in $S_{1}$ $\left(\tilde{\nu}_{v}=400 \mathrm{~cm}^{-1}[22]\right)$. The lower intensity of this hot band at $110 \mathrm{~K}$ (relative to the 0,0 transition) is roughly in accord with a delayed fluorescence from thermally equilibribrated molecules in $S_{1}$.

As a consequence of the lower relative intensity of equilibrium hot-band delayed $S_{1} \rightarrow S_{0}$ fluorescence, a larger fraction of the $S_{2} \rightarrow S_{0}$ band of the delayed fluorescence can be seen. Apart from the origin band at $29500 \mathrm{~cm}^{-1}$, the first strong vibronic contour band of the delayed $S_{2} \rightarrow S_{0}$ fluorescence at $28200 \mathrm{~cm}^{-1}$ is observed. In this respect a lower temperature (110 $\mathrm{K})$ has roughly the same effect as selective quenching of the delayed $S_{1} \rightarrow S_{0}$ fluorescence at $193 \mathrm{~K}$ [37].

The origin band itself has at $110 \mathrm{~K}$ some structure (shoulder at $29100 \mathrm{~cm}^{-1}$ ), which is not yet discernible in the spectrum at $193 \mathrm{~K}$. The loss of structure in the spectrum of delayed $S_{2} \rightarrow S_{0}$ fluorescence relative to the $S_{0} \rightarrow S_{2}$ absorption spectrum has two causes: (1) Sequence broadening resulting from the fact that the delayed $S_{2} \rightarrow S_{0}$ fluorescence is emitted by vibrationally unrelaxed molecules, and (2) additional broadening resulting from interaction between the excited molecule and the ground-state molecule in the $S_{2} \ldots S_{0}$ pair emerging from TTA [2]. An increase of the average distance $\bar{R}$, at which TTA takes place, leads to a weaker broadening resulting from interaction in the molecular pairs. In section 5.1 .3 it is shown that indeed $\bar{R}$ is in isopentane/cyclopentane at $110 \mathrm{~K}$ higher than in methylcyclohexane at $193 \mathrm{~K}$. An analogous argument applies to the structure in the spectral range (g).

\subsubsection{Delayed excimer fluorescence from pyrene}

The intensity ratio $I_{\mathrm{DFE}} / I_{\mathrm{DF} 1}$ of delayed excimer fluorescence and delayed $S_{1}$ monomer fluorescence from pyrene is in isopentane/cyclopentane at $110 \mathrm{~K}$ (fig. 2) lower by a factor of $\approx 0.5$ than in methylcyclohexane at $193 \mathrm{~K}$ (fig. 1 ). The viscosity of isopentane/cyclopentane at $110 \mathrm{~K}(\approx 100 \mathrm{cP})$ is $\approx 10$ times higher than that of methylcyclohexane at $193 \mathrm{~K}(\approx 10$ cP). At low temperatures, where excimer dissociation is negligible and the lifetime of $S_{1}$ and the quantum yield of prompt $S_{1} \rightarrow S_{0}$ fluorescence of isolated molecules (in contrast to the molecular pairs in TTA) are nearly temperature independent, a decrease of $I_{\mathrm{DFE}} / I_{\mathrm{DF} 1}$ with decreasing temperature can have two causes: (1) The excimer is formed with decreasing probability. (2) The quantum yield of the excimer fluorescence decreases. Since there is no indication of the effectiveness of the second cause [36], one may safely assume that only the first cause is responsible for the observed decrease of $I_{\mathrm{DFE}} / I_{\mathrm{DF} 1}$.

In general, the probability of excimer formation after TTA must decrease with decreasing ratio $T / \eta$ of temperature $T$ and viscosity $\eta$ on two grounds: Firstly, the average distance $\vec{R}$ at which TTA takes place depends on the distance dependence $k_{\mathrm{nr}}(R)$ of the firstorder rate constant $k_{\mathrm{nr}}$ of nonradiative TTA in an $T_{1} \ldots T_{1}$ encounter pair and on the rate at which translational and rotational diffusion takes place [28]. A decrease of $T / \eta$ will increase $\bar{R}$ and thus also increase the probability of dissociation of the $S_{1} \ldots S_{0}$ pair formed by TTA (dissociation as a competitive process to excimer formation ). Secondly, a decrease of $T / \eta$ means a slower translational and rotational diffusion and thus a reduced probability of transformation of an $S_{1} \ldots S_{0}$ pair into an excimer. We estimate 
that the orientation relaxation time of pyrene in isopentane/cyclopentane at $110 \mathrm{~K}$ is of the order of 10 ns $[21,25]$, that means, much shorter than the lifetime of $S_{1}(\approx 500 \mathrm{~ns}$ in isolated molecules [36]). Therefore we conclude that the observed decrease of $I_{\mathrm{DFE}} / I_{\mathrm{DF} 1}$ in isopentane/cyclopentane must result nearly exclusively from an increase of $\bar{R}$.

The spectra of the delayed excimer fluorescence in figs. 1 and 2 are not identical. The excimer band in fig. 2 is narrower and slightly red-shifted relative to the excimer band in fig. 1 . The difference is mainly a temperature effect and not a solvent effect. To illustrate this point, we show in fig. 5 the temperature dependence of the spectrum of the excimer fluorescence from pyrene in isopentane ${ }^{\# 1}$. We have not been able to reproduce the definite onset of the excimer fluorescence reported in ref. [2]. The absence of a definite onset of the excimer band is, however, no proof of the excimer fluorescence being a transition from a bound excited dimer to an unbound groundstate dimer ${ }^{\# 1}$.

\#1 The spectra in fig. 5 were measured by Dr. M.F. Rodriguez Prieto in our laboratory with the aim to test a simple excimer model. We intend to treat this model in another paper.

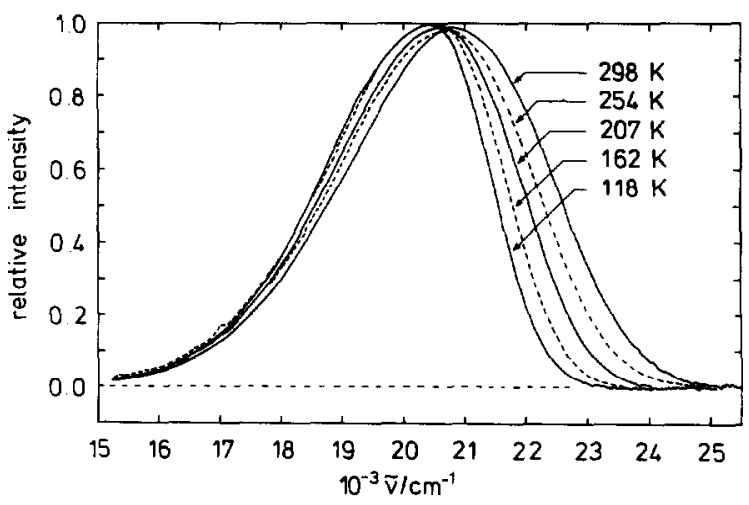

Fig. 5. Temperature dependence of the corrected excimer fluorescence spectrum of pyrene in isopentane. The spectra at 118 , 162 and $207 \mathrm{~K}$ are difference spectra of the spectra of delayed fluorescence and prompt fluorescence of a $5 \times 10^{-6} \mathrm{M}$ solution of pyrene. The spectra at 254 and $298 \mathrm{~K}$ are difference spectra of the prompt fluorescence at $5 \times 10^{-4}$ and $5 \times 10^{-6} \mathrm{M}$ pyrene. The excitation wavelength was $333 \mathrm{~nm}$ for the low concentration and $351 \mathrm{~nm}$ for the high concentration of pyrene.

\subsection{Delayed luminescence from phenazine}

In fig. 6 we show the spectrum of the delayed luminescence from a liquid solution of phenazine in isopentane/cyclopentane $(7: 3)$ at $110 \mathrm{~K}$. Phenazine is a strongly phosphorescent and weakly fluorescent compound. Hence the phosphorescence from phenazine in fluid solution is easy to observe (the relative intensity of the phosphorescence increases with decreasing triplet concentration). The phosphorescence spectrum is shown separately and in a larger spectral range in fig. 7. From left to right, the delayed-luminescence spectrum in fig. 6 consists of the following components:

(a) $\tilde{\nu} \leqslant 15900 \mathrm{~cm}^{-1}:$ structured phosphorescence;

(b) $15900 \mathrm{~cm}^{-1} \lesssim \tilde{\nu} \lesssim 16400 \mathrm{~cm}^{-1}$ : dominant hotband phosphorescence;

(c) $16400 \mathrm{~cm}^{-1} \lesssim \tilde{\nu} \leqslant 23000 \mathrm{~cm}^{-1}$ : nearly structureless delayed monomer-like $S_{1} \rightarrow S_{0}$ fluorescence;

(d) $23000 \mathrm{~cm}^{-1} \leqslant \tilde{\nu} \leqslant 24500 \mathrm{~cm}^{-1}$ : dominant nonequilibrium hot bands of delayed $S_{1} \rightarrow S_{0}$ fluorescence;

(e) $24500 \mathrm{~cm}^{-1} \leqslant \nu \leqslant 27500 \mathrm{~cm}^{-1}$ : delayed monomer-like $S_{2} \rightarrow S_{0}$ and $S_{3} \rightarrow S_{0}$ fluorescence;

(f) $27500 \mathrm{~cm}^{-1} \leqslant \tilde{\nu} \leqslant 29000 \mathrm{~cm}^{-1}$ : dominant nonequilibrium hot bands of delayed $S_{3} \rightarrow S_{0}$ fluorescence;

(g) $29000 \mathrm{~cm}^{-1} \leqslant \tilde{\nu} \leqslant 31400 \mathrm{~cm}^{-1}$ : an extremely weak delayed luminescence near $2 E\left(T_{1,0}\right)$.

Since the observation of delayed fluorescence from phenazine is new, we first justify the assignment of the spectral ranges (c)-(f) and then compare the high-energy region $(g)$ with the calculated spectrum of radiative TTA.

\subsubsection{Delayed fluorescence from phenazine}

In the absorption spectrum of phenazine in the visible and the near UV, three transitions [38] can be distinguished:

(1) A very weak $S_{0} \rightarrow S_{1}\left(n \pi^{*}, B_{1 u}\right)$ transition polarized in the $z$ axis (perpendicular to the plane of the molecule) and with origin at $\approx 23000 \mathrm{~cm}^{-1}(22243$ $\left.\mathrm{cm}^{-1}\right)$.

(2) A weak $\mathrm{S}_{0} \rightarrow \mathrm{S}_{2}\left(\mathrm{~L}_{\mathrm{a}}, \mathrm{B}_{3 \mathrm{u}}\right)$ transition polarized in the $x$ axis (short axis of the molecule) and with origin at $\approx 26000 \mathrm{~cm}^{-1}\left(25404 \mathrm{~cm}^{-1}\right)$.

(3) A moderately strong $S_{0} \rightarrow S_{3}\left(L_{b}, B_{2 u}\right)$ transition polarized in the $y$ axis (long axis of the molecule) and with origin at $\approx 27500 \mathrm{~cm}^{-1}(27502$ $\mathrm{cm}^{-1}$ ). 


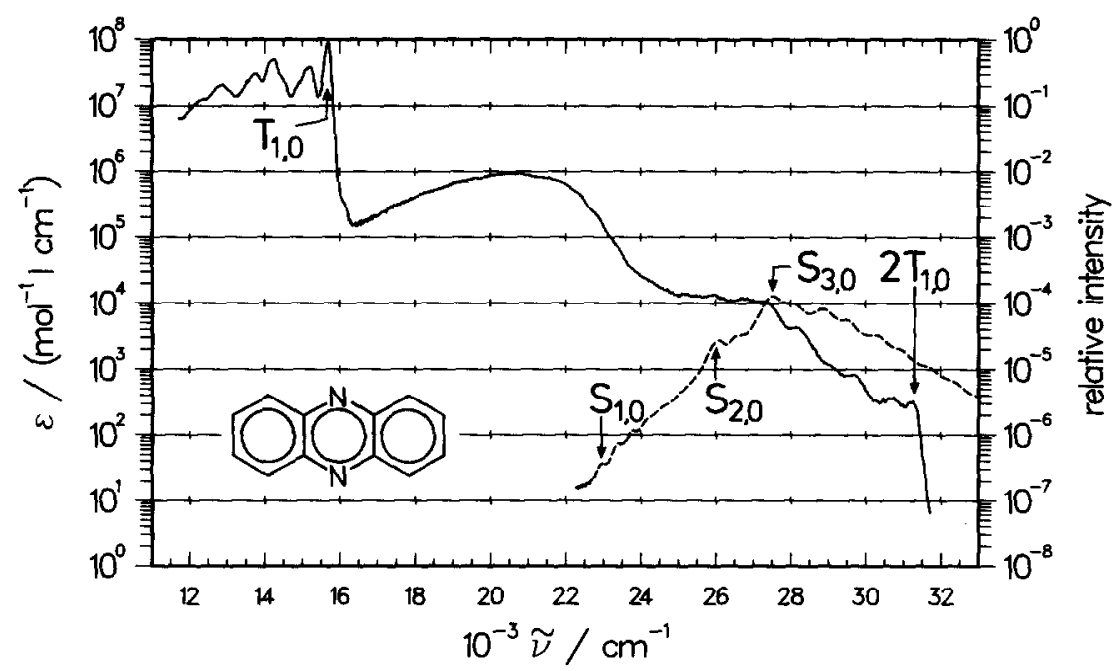

Fig. 6. Spectrally corrected spectrum of the delayed luminescence (-) from a solution of $2.0 \times 10^{-5} \mathrm{M}$ phenazine in isopentane/ cyclopentane $(7: 3)$ at $110 \mathrm{~K}$ and absorption spectrum $\epsilon(\tilde{\nu})(---)$ of phenaxine at the same temperature. Phenazine was excited with the $363.8 \mathrm{~nm}$ UV line of an argon ion laser. The measurement of region ( $\mathrm{g}$ ) of the delayed-luminescence spectrum took about one day. Reabsorption of delayed luminescence in the sample did not exceed 5\% in the high-energy region ( $\mathrm{g}$ ) above $29000 \mathrm{~cm}^{-1}$.

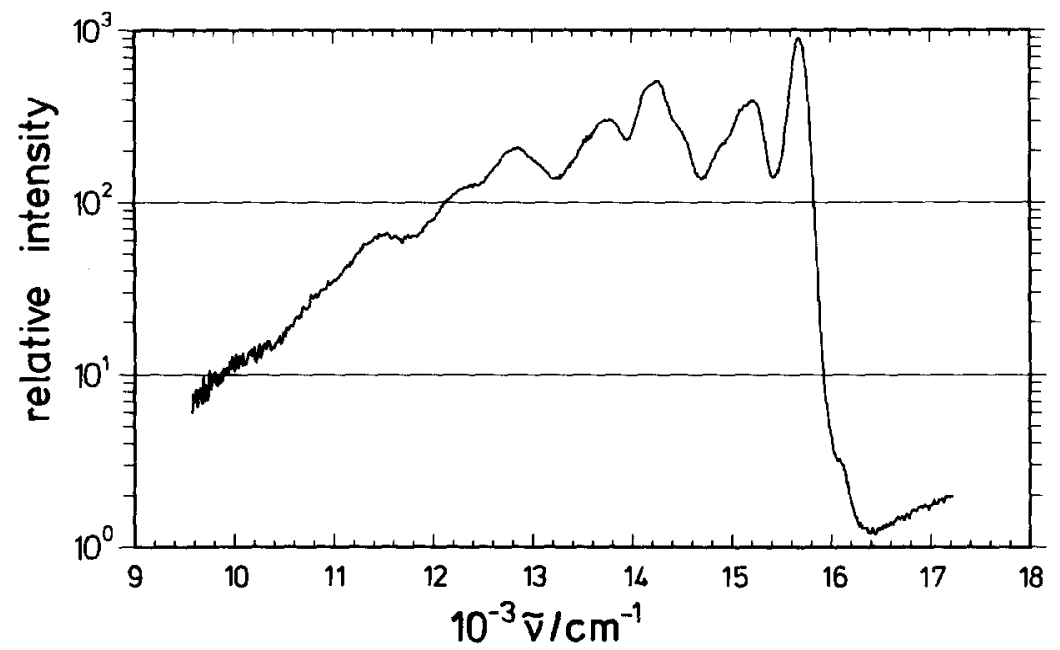

Fig. 7. Spectrally corrected spectrum of the phosphorescence from a solution of $2.0 \times 10^{-5} \mathrm{M}$ phenazine in isopentane/cyclopentane $(7: 3)$ at $110 \mathrm{~K}$.

The numbers in parentheses give the origins found by Narva and McClure [38] with phenazine in a biphenyl host crystal. For the very weak $S_{1}$ transition a difference in the origin wavenumbers of $\approx 750 \mathrm{~cm}^{-1}$ seems to be unusually large. Possibly the band at
$23000 \mathrm{~cm}^{-1}$ in the absorption spectrum of phenazine in 3-methylpentane is not the true origin but the first strong vibronic contour band $\approx 400 \mathrm{~cm}^{-1}$ above the true origin.

The delayed luminescence in the spectral range (c) 
can be interpreted as delayed monomer-like $S_{1} \rightarrow S_{0}$ fluorescence. The spectrum is very similar to the nearly structureless spectrum of the prompt fluorescence from phenazine in hexane at room temperature with a maximum at $20500 \mathrm{~cm}^{-1}$ and with a quantum yield $\phi_{\mathrm{F} 1}=(3.0 \pm 0.7) \times 10^{-5}[39]$.

For the following discussion a reliable value of the lifetime $\tau_{1}$ of $S_{1}$ of phenazine is important. Hirata and Tanaka [40] obtained from transient-absorption measurements $\tau_{1} \approx 14$ ps for phenazine in isooctane. Lin and Topp [41] measured the fluorescence from upper excited singlet states of phenazine in hexane by consecutive $S_{0} \rightarrow S_{1} \rightarrow S_{n}$ excitation of phenazine; from the decreasing intensity of the upper-state fluorescence with increasing delay of the second excitation light pulse they obtained $\tau_{1}=62 \pm 5$ ps. For two reasons we consider the second value of $\tau_{1}$ as the more reliable one. Firstly, the transient-absorption spectrum measured 50 ps after excitation [40] shows significant deviations from published triplet-triplet absorption spectra of phenazine [42] (for a recent compilation of triplet-triplet absorption spectra of phenazine cf. ref. [43]), and Hirata and Tanaka do not discuss to what extent the observed transient absorption may be due to $S_{l} \rightarrow S_{n}$ absorption. Secondly, the greater value of $\tau_{1}$ yields a longer radiative lifetime $\tau_{\mathrm{F} 1}=\tau_{1} / \phi_{\mathrm{F} 1} \approx 2 \mu \mathrm{s}$, which is in better agreement with the radiative lifetime estimated from the very weak $\mathbf{S}_{0} \rightarrow \mathbf{S}_{1}$ absorption band.

From $\tau_{1} \approx 60$ ps we draw four conclusions: Firstly, since lifetimes of upper excited singlet states $S_{n}$ are typically much shorter than $60 \mathrm{ps,} \mathrm{internal} \mathrm{conver-}$ sion $S_{n}{ }^{m} S_{1}$ is likely to be the dominant nonradiative decay process of $S_{n}$ (and not intersystem crossing $S_{n} \leadsto T_{1}$ or internal conversion $S_{n} \leadsto S_{0}$ ). Secondly, we assume that the $S_{1} \rightsquigarrow T_{1}$ intersystem crossing is the only fast decay process of $S_{1}$ and that its rate constant is nearly independent of temperature (as in other cases of molecules with an $S_{1}\left(n \pi^{*}\right)$ state). This assumption is in accord with the reported independence of $\phi_{\mathrm{F} 1}$ on temperature [39]. Thirdly, under the given experimental conditions, orientational relaxation of molecules $S_{1}$ is slow relative to the decay of $S_{1}$. Therefore, excimer formation following TTA can be neglected ${ }^{\# 2}$, in accord with the observed similarity of the spectra of prompt and delayed fluorescence. Fourthly, since internal conversion $S_{n} \leftrightarrow S_{1}$ leads to vibrationally excited molecules in $S_{1}$, and since vibrational relaxation times of large molecules in condensed phase are typically in the range between 10 and 100 ps [44-48], a rather high relative intensity of nonequilibrium hot-band delayed fluorescence from $S_{1}$ is expected and indeed observed - see spectral range (d). In conclusion, we believe that the intensity of the delayed fluorescence from $S_{1}$ can be used as reference intensity for an estimate of the relative probability of radiative TTA in section 5.2.2.

The delayed luminescence in the spectral range (e) is assigned to delayed fluorescence from $S_{2}$ and $S_{3}$ : The two maxima at 27300 and $26700 \mathrm{~cm}^{-1}$ are mirror symmetric to the origin at $27500 \mathrm{~cm}^{-1}$ and the first vibronic contour band at $28100 \mathrm{~cm}^{-1}$ of the $S_{3}$ absorption band, and the weak maximum at 25900 $\mathrm{cm}^{-1}$ is only slightly red-shifted relative to the origin of the $S_{2}$ absorption band at $25000 \mathrm{~cm}^{-1}$ and symmetric to the second $S_{3}$ vibronic contour band at $28850 \mathrm{~cm}^{-1}$.

The total intensity of the delayed $S_{3} \rightarrow S_{0}$ fluorescence is roughly by a factor of $10^{-2}$ weaker than that of the delayed $S_{1} \rightarrow S_{0}$ fluorescence; the corresponding fluorescence quantum yield is $\phi_{\mathrm{F} 3} \approx 3 \times 10^{-7}$. This very low value may have several causes. Firstly, as in the case of $S_{2}$ of pyrene, the vibronic states resulting from strong intermediate $S_{3}-S_{2}-S_{1}$ vibronic coupling are only weakly fluorescent (cf. section 5.7). Secondly, internal conversion of these vibronic states into pure $S_{1}$ vibronic states may be very fast. Thirdly, according to an INDO/SDCI calculation [49] of electronically excited singlet and triplet states of phenazine, the state $S_{4}\left(n \pi^{*}, B_{2 g}\right)$ should be accessible by TTA. Hence dominant primary population of $S_{4}$ by TTA and effective direct internal conversion $S_{4} \leftrightarrow S_{1}$ may be also responsible for a low relative quantum yield of delayed fluorescence from $S_{2}$ and $S_{3}$. Finally we note that upper-state delayed fluorescence with a quantum yield of the order of $3 \times 10^{-7}$ is not unique. For example, biphenylene has an $S_{0} \rightarrow S_{2}$ absorption

\#2 If phenazine in principle does form an excimer, then a delayed excimer fluorescence from phenazine in isopentane/cyclopentane should be observable at higher temperatures and lower viscosity, where the orientational relaxation time is shorter than the lifetime of $S_{1}$. 
band with an oscillator strength similar to that of the $\mathrm{S}_{0} \rightarrow \mathrm{S}_{3}$ absorption band of phenazine, and the quantum yield of the delayed fluorescence from $\mathrm{S}_{2}$ of biphenylene is $\approx 2 \times 10^{-7}[25]$.

\subsubsection{High-energy region of the delayed luminescence from phenazine}

In fig. 8 the high-energy region $(\mathrm{g})$ of the delayedluminescence spectrum $I_{\mathrm{DL}}(\tilde{\nu})$ is compared with two calculated spectra $I_{\mathrm{rad}}^{(1)}(\tilde{\nu})$ and $I_{\mathrm{rad}}^{(2)}(\tilde{\nu})$ of radiative TTA. The spectra were calculated from the phosphorescence spectrum in fig. 7 with eq. (11) and with a red shift $\Delta \tilde{\nu}_{\mathrm{TT}}=130 \mathrm{~cm}^{-1}$ and the Gaussian widths $B(1)=0 \mathrm{~cm}^{-1}$ and $B E_{G}^{(2)}=150 \mathrm{~cm}^{-1}$. In the spectral range between 29000 and $31320 \mathrm{~cm}^{-1}\left(2 E\left(\mathrm{~T}_{1,0}\right)\right)$, $I_{\mathrm{DL}}(\tilde{\nu})$ and $I_{\mathrm{rad}}^{(2)}(\tilde{\nu})$ have the same structure, and differences between these spectra do not exceed $\pm 15 \%$. Therefore, on the same grounds as in the case of pyrene (cf. section 5.1.1), the dominant component of the delayed luminescence in this spectral range can be assigned to radiative TTA. Note that, as pyrene, phenazine is also a centrosymmetric molecule. Hence the observation of a delayed luminescence at $\approx 2 E\left(T_{1,0}\right)$ is an additional argument in favour of this assignment.

With the assumption that radiative TTA is the dominant delayed luminescence in the spectral range (g), the relative probability $k_{\mathrm{r}} / k_{\mathrm{nr}}$ of radiative TTA can be estimated. We assign the delayed luminescence at $30600 \mathrm{~cm}^{-1}$ completely to radiative TTA. With $\phi_{\mathrm{Fl}} \approx 3 \times 10^{-5}, I_{\mathrm{DFE}}=0$, and $p_{\mathrm{rad}} \approx 0.7$, eqs. (15) and (16) yield $k_{\mathrm{r}} / k_{\mathrm{nr}} \approx 3 \times 10^{-8}$.

Originally we had expected that there might be a correlation between the rate constant $k_{\mathrm{r}}$ of radiative TTA and the rate constant $k_{\mathrm{P}}$ of unimolecular radiative triplet decay ( $=$ phosphorescence). In this respect the very low value of $k_{\mathrm{r}} / k_{\mathrm{nr}}$ was disappointing. However, the observed low value of $k_{\mathrm{r}} / k_{\mathrm{nr}}$ does not rule out a correlation between $k_{\mathrm{r}}$ and $k_{\mathrm{P}}$. We again regard nonradiative TTA as a special case of Dexter energy transfer and expect an unusually high value of $k_{\mathrm{nr}}$, since the two spin-forbidden transitions $\mathrm{T}_{1} \rightarrow \mathrm{S}_{0}$ and $T_{1} \rightarrow S_{n}$ in the singlet channel of nonradiative TTA are in phenazine much stronger than in a typical aromatic hydrocarbon like pyrene. This point is further discussed in section 5.6.2.

\subsection{Delayed luminescence from anthracene}

We measured the delayed-luminescence spectrum and the absorption spectrum of anthracene in isopentane/cyclopentane at $\approx 110 \mathrm{~K}$ (see fig. 9). The

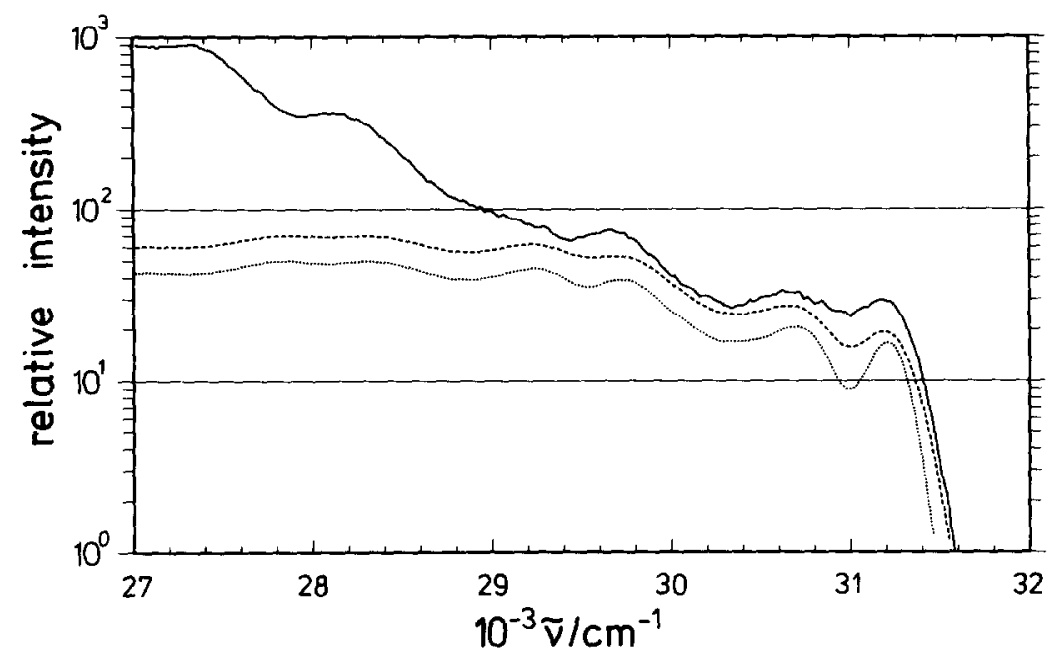

Fig. 8. Comparison of the high-energy region $(\mathrm{g})$ of the delayed-luminescence spectrum of phenazine in fig. 6 ( - ) with two spectra $I_{\mathrm{rad}}(\tilde{\nu})$ of radiative TTA calculated from the phosphorescence spectrum in fig. 7 with $\Delta \tilde{\nu}_{\mathrm{TT}}=130 \mathrm{~cm}^{-1}$ and two different values of $B_{G}: I_{\mathrm{rad}}^{(1)}(\tilde{\nu})$ with $B g^{(1)}=0 \mathrm{~cm}^{-1}(\ldots)$ and $I_{\mathrm{rad}}^{(2)}(\tilde{\nu})$ with $B G^{(2)}=150 \mathrm{~cm}^{-1}(-)$. The three spectra are vertically shifted relative to each other. 


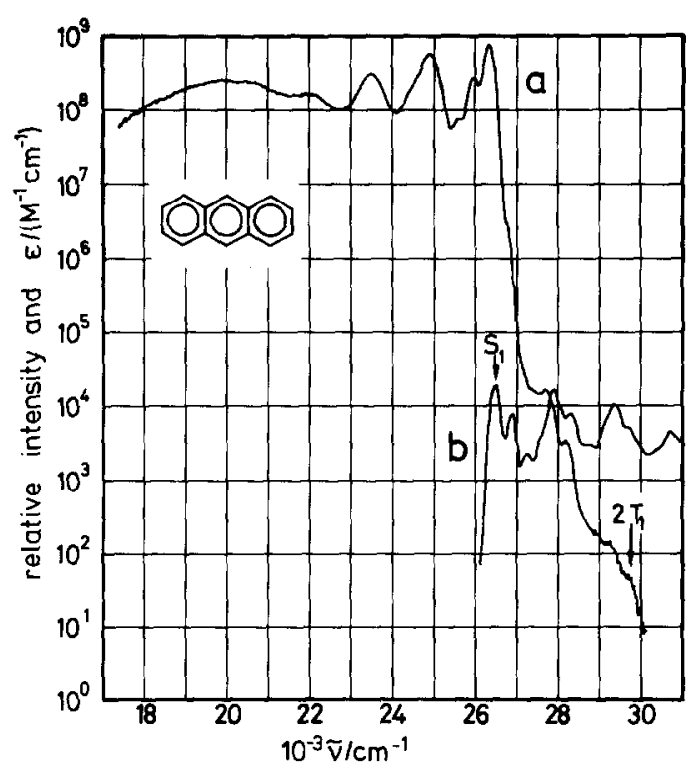

Fig. 9. (a) Spectrally corrected spectrum of the delayed luminescence from a solution of $2.0 \times 10^{-5} \mathrm{M}$ anthracene in isopentane/ cyclopentane $(7: 3)$ at $112 \mathrm{~K}$ and (b) absorption spectrum $\epsilon(\tilde{v})$ of anthracene at $110 \mathrm{~K}$. Anthracene was excited with all lines of the argon ion laser in the near UV $(364,351$ and $333 \mathrm{~nm})$.

spectrum of the delayed luminescence consists of four distinct spectral ranges:

(a) $17400 \mathrm{~cm}^{-1} \lesssim \tilde{\nu} \lesssim 21500 \mathrm{~cm}^{-1}$ : dominant delayed excimer fluorescencc;

(b) $21500 \mathrm{~cm}^{-1} \lesssim \tilde{\nu} \leqslant 26500 \mathrm{~cm}^{-1}$ : delayed monomer-like $S_{1} \rightarrow S_{0}$ fluorescence;

(c) $26500 \mathrm{~cm}^{-1} \leqslant \tilde{\nu} \leqslant 29500 \mathrm{~cm}^{-1}$ : hot bands of delayed monomer-like $S_{1} \rightarrow S_{0}$ fluorescence;

(d) $\tilde{\nu} \approx 29700 \mathrm{~cm}^{-1}$ : an extremely weak delayedluminescence band at roughly the wavenumber corresponding to $2 E\left(\mathrm{~T}_{1,0}\right)\left(29760 \mathrm{~cm}^{-1}\right)$.

The assignment of the spectral ranges (a) and (b) is obvious and needs no further comment. The assignment of the spectral range (c) follows from the comparison of the structure of the delayed-luminescence spectrum with that of the absorption spectrum: To each shoulder or maximum in delayed luminescence there is a counterpart in absorption. This is shown more clearly in table 1 . We discuss the different bands in turn.

The relative intensity of band no. 1 is roughly in accord with an equilibrium population of the vibronic state $S_{1, v_{1}}\left(\tilde{\nu}_{v_{1}} \approx 400 \mathrm{~cm}^{-1}\right)$ relative to $S_{1,0}$.
Band no. 2 takes an intermediate position. Band no. 3 and all following bands are stronger than expected for an equilibrium population of $S_{1, \nu_{i}}$.

If one assumes that the bands no. 4 to no. 8 represent delayed fluorescence from the vibronic states generated by TTA, then one can show that the order of magnitude of the relative intensity of these bands is reasonable. In the following we derive an expression for the relative intensity of vibronic contour bands of non-equilibrium hot-band delayed fluorescence from $S_{1}$.

Let $S_{1, i j}$ be a vibronic state, where $i$ and $j$ stand for single vibrations or combinations of vibrations. Let the set $\left\{S_{1, i j} \rightarrow S_{0,0, j}\right\}$ of overlapping vibronic transitions be observed in the spectrum of the delayed fluorescence in the form of the single vibronic contour band no. $i$. The intensity of these vibronic transitions is approximately given by

$I_{\mathrm{DF}}^{i \rightarrow 0}=C\left(\sum_{j} p_{i j} \tau_{i j}\right) k_{\mathrm{F}}^{i \rightarrow 0}$

where $C$ is a constant, $p_{i j}$ is the probability for the creation of $S_{1, i, j}$ by TTA, and $\tau_{i j}$ is the lifetime of the vibration $i$. (Note that the lifetime $\tau_{i j}$ can be longer than the lifetime of $S_{1, i j}$, since the intramolecular redistribution of vibrational energy $S_{1, i, j}{ }^{m+} S_{1, i, j}$ with $j^{*} \neq j$ represents a decay of $S_{1, i j}$ but does not necessarily affect the intensity of the band no. $i$ of the delayed fluorescence.) The radiative-decay rate constant $k_{\mathrm{F}}^{i \rightarrow 0}$ is assumed to be independent of $j$. In order to apply eq. (17), we have to make further simplifying assumptions:

(1) We replace $\sum_{j} p_{i j} \tau_{i j}$ by $p_{i} \tau_{i}$, where $p_{i}$ is the total probability for the generation of vibronic states $S_{1, i, j}$ containing one or more quanta of the vibration no. $i$ and $\tau_{i}$ is the average lifetime of the vibration $i$.

(2) We assume that $k_{\mathrm{F}}^{i \rightarrow 0}$ is proportional to the maximum of the molar absorption coefficient $\epsilon_{0 \rightarrow i}$ of the inhomogeneously broadened vibronic transition $\mathbf{S}_{0,0} \rightarrow \mathbf{S}_{1, i}$ :

$I_{\mathrm{DF}}^{i \rightarrow 0}=C^{\prime} p_{i} \tau_{i} \epsilon_{0 \rightarrow i}$

(3) One has to account for the possibility that several different vibronic transitions $S_{0,0} \rightarrow S_{1, i_{k}}(k=1$, $\left.2, \ldots, n_{i}\right)$ are hidden under the vibronic contour band no. $i$ : 
Table 1

Assignment of vibronic contour bands in the spectrum of the hot-band delayed luminescence from anthracene to vibronic contour bands in the absorption spectrum of anthracene - see fig. 9. Abbreviations: m: maximum, s: shoulder, ws: weak shoulder

\begin{tabular}{|c|c|c|c|c|c|}
\hline No. & $\begin{array}{l}\text { Del. lumin. } \\
\left(\mathrm{cm}^{-1}\right)\end{array}$ & Type & Intensity & $\begin{array}{l}\text { Absorption } \\
\left(\mathrm{cm}^{-1}\right)\end{array}$ & Type \\
\hline 0 & 26340 & $\mathrm{~m}$ & 1 & 26490 & $\mathbf{m}$ \\
\hline 1 & 26720 & $\mathbf{s}$ & $3.9 \times 10^{-3}$ & 26880 & $\mathrm{~m}$ \\
\hline 2 & 27180 & ws & $3.5 \times 10^{-3}$ & 27235 & $\mathbf{m}$ \\
\hline 3 & 27405 & $\mathbf{s}$ & $2.2 \times 10^{-5}$ & 27550 & $s$ \\
\hline 4 & 27705 & m & $2.2 \times 10^{-5}$ & 27905 & $\mathrm{~m}$ \\
\hline 5 & 28165 & $\mathrm{~m}$ & $4.4 \times 10^{-6}$ & 28310 & $\mathbf{m}$ \\
\hline 6 & 28600 & ws & $4.4 \times 10^{-7}$ & 28745 & $\mathrm{~m}$ \\
\hline 7 & 28965 & $\mathbf{s}$ & $2.4 \times 10^{-7}$ & 29115 & $s$ \\
\hline 8 & 29235 & m & $1.8 \times 10^{-7}$ & 29370 & $\mathrm{~m}$ \\
\hline 9 & 29700 & $\mathrm{~s}$ & $6.4 \times 10^{-8}$ & 29715 & $\mathrm{~s}$ \\
\hline $2 T_{1,0}$ & 29760 & & & & \\
\hline
\end{tabular}

$I_{\mathrm{DF}}^{i \rightarrow 0}=C^{\prime} \sum_{k=1}^{n_{i}} p_{i k} \tau_{i k} \epsilon_{0 \rightarrow i k}$

(4) Finally, with the assumption that the $n_{i}$ overlapping vibronic transitions $i_{k}$ have similar strength, eq. (19) reduces to

$I_{\mathrm{DF}}^{i \rightarrow 0} \approx C^{\prime} p_{i} \tau_{i} \epsilon_{0 \rightarrow i} / n_{i}$,

where $p_{i}$ is now the sum of the $p_{i k}, \tau_{i}$ is the average lifetime of the vibrations $i_{k}$, and $\epsilon_{0 \rightarrow i}$ is the molar extinction coefficient in the maximum of the $i$ th vibronic contour band.

The relative intensity $R_{0}^{i}$ of the $i$ th vibronic contour band of the delayed fluorescence can now be written in the form

$R_{0}^{i} \approx\left(p_{i} / p_{0}\right)\left(\tau_{i} / \tau_{0}\right)\left(\epsilon_{0 \rightarrow i} / \epsilon_{0 \rightarrow 0}\right) n_{i}^{-1}$.

We now apply eq. (21) to band no. 4 (cf. table 1 ). For an estimate of $p_{4}$ we assume that in the nonradiative TTA $T_{1,0}+T_{1,0} * S_{1, v}+S_{0, w}$ the excess energy

$\Delta E=2 E\left(T_{1,0}\right)-E\left(S_{1,0}\right) \approx h c \times 3400 \mathrm{~cm}^{-1}$

reappears as vibrational energy with equal probability in $S_{1, v}$ and $S_{0, w}$. Thus the average vibrational energy $\left\langle E_{\nu}\right\rangle$ in $S_{1, v}$ should be $\left\langle E_{v}\right\rangle \approx h c \times 1700 \mathrm{~cm}^{-1}$. Since $S_{1, v}$ should contain one quantum of a $u$ vibration (cf. section 2.2), we estimate the average amount of vibrational energy in the form of $g$ vibrations to be $\left\langle E_{\nu_{\mathrm{g}}}\right\rangle \approx h c \times 1500 \mathrm{~cm}^{-1}$. If in all states $S_{1, i, j}$ with $E_{v_{\mathbf{g}}} \geqslant\left\langle E_{v_{\mathbf{g}}}\right\rangle$ one of the vibrations of the vibronic absorption contour band no. 4 were excited, we would have $\left(p_{4}\right)_{\max } \approx 0.5$. Hence we assume $0.1 \lesssim p_{4} \lesssim 0.5$. For the determination of $p_{0}$ one may assume that the deactivation of all vibronic states $S_{1, i, j}$ leads to $S_{1,0}$ (Vavilov's rule); if only delayed monomer fluorescence were observable, one would have $p_{0}=1$. We take the delayed excimer fluorescence into account by choosing $p_{0} \approx 0.7$.

For an estimate of $\tau_{4}$, the following literature data can be used: (1) The prompt nonequilibrium hotband fluorescence from anthracene in different matrices at very low temperatures has been investigated [50]. The indirectly determined vibronic lifetimes are $2.5 \pm 1.0 \mathrm{ps}$ for $\tilde{\nu}_{v}=1400 \mathrm{~cm}^{-1}$ and $5 \pm 2 \mathrm{ps}$ for $\tilde{\nu}_{v}=1500 \mathrm{~cm}^{-1}[50]$. (2) The time-resolved measurement of the hot fluorescence with $\tilde{\nu}_{v}=1400 \mathrm{~cm}^{-1}$ yielded the vibronic lifetime $19 \pm 3$ ps [51]. (3) For anthracene in the electronic ground state and $\tilde{\nu}_{v}=$ $1403 \mathrm{~cm}^{-1}$, with time-resolved spontaneous antiStokes Raman scattering a vibrational lifetime of $10 \pm 5$ ps was obtained [52]. From these data we obtain 2 ps $\lesssim \tau_{4} \leqslant 10$ ps for the average lifetime of the vibronic states $S_{1, \nu_{\mathrm{g}}}$ observed in the absorption spectrum at $\approx 1400 \mathrm{~cm}^{-1}$ above the origin. For an estimate of $\tau_{0}$ we assume that the radiative lifetime $\tau_{F 1}$ of $S_{1}$ does not appreciably depend on solvent and temperature. Then with $\tau_{\mathrm{Fl}} \approx 17 \mathrm{~ns}$ (anthracene in cyclohexane at room temperature, table 4.3 in ref. [36]) and the fluorescence quantum yield $\phi_{F 1}=0.70$ (extrapolated value for anthracene in heptane at 110 $\mathrm{K}$ [53]), follows $\tau_{0}=\tau_{\mathrm{F} 1} \phi_{\mathrm{F} 1} \approx 12 \mathrm{~ns}$.

From the absorption spectrum in fig. 9 follows 
$\epsilon_{0 \rightarrow 4} / \epsilon_{0 \rightarrow 0} \approx 1$. For an estimate of $n_{4}$ we refer to the absorption spectrum of isolated anthracene molecules in a supersonic jet $[54,55]$; from this spectrum follows that there are $n_{4}=4$ vibronic transitions of similar strength between 1380 and $1514 \mathrm{~cm}^{-1}$ above $\mathrm{S}_{1,0}$.

With the preceding estimated parameter values, eq. (21) yields $6 \times 10^{-6} \leqq R_{0}^{4} \leqq 1.5 \times 10^{-4}$ - in satisfactory agreement with the experimental value $2.2 \times 10^{-5}$ in table 1 . In a similar way the relative intensity of band no. 8 can be understood. For example, the following set of parameters would yield the experimental values $R_{0}^{8} \approx 1.8 \times 10^{-7}: p_{8}=0.05, \tau_{8}=1$ ps, $\epsilon_{0 \rightarrow 8} / \epsilon_{0 \rightarrow 8}=0.5$, and $n_{8}=20$. (Note that, because of the great uncertainty of the value of $p_{8}, \tau_{8}$ and $n_{8}$, the uncertainty of an estimate of $R_{0}^{8}$ is at least of the order of $10^{ \pm 1}$.) In conclusion, as far as vibrational structure and the order of magnitude of vibronic intensities are concerned, the whole spectral range (c) can be assigned to delayed fluorescence from anthracene.

The only band left for the assignment to radiative TTA is the band no. 9 at roughly the energy $2 E\left(T_{1,0}\right)$. The main argument for this assignment is that according to the symmetry considerations of section 2.2 no delayed fluorescence should be observable at $2 E\left(\mathrm{~T}_{1,0}\right)$. Conversely, if radiative TTA is the dominant contribution to band no. 9 , it cannot be the dominant contribution to band no. 8 , in accord with the assignment of band no. 8 to delayed fluorescence. This follows from the structure of the phosphorescence spectrum of anthracene. Though only the uncorrected phosphorescence spectrum is known [56], it is clear that the first strong vibronic contour band is at $\approx 1400 \mathrm{~cm}^{-1}$ below $E\left(\mathrm{~T}_{1,0}\right)$. According to eq. (11) that means, the spectrum of radiative TTA cannot have a strong band $\approx 500 \mathrm{~cm}^{-1}$ below $2 E\left(T_{1,0}\right)$, at the wavenumber of band no. 8 .

The relative probability $k_{\mathrm{r}} / k_{\mathrm{nr}}$ of radiative TTA cannot be estimated in the same way as before, since the calculated spectrum of radiative TTA is not known. However, if we assume an intensity distribution similar to that of the spectrum of radiative TTA of phenazine, we obtain $k_{\mathrm{r}} / k_{\mathrm{nr}} \approx 10^{-7}$, in rough agreement with the order-or-magnitude estimates in refs. [5-7].

Finally we have to justify the neglect of the $S_{2}\left(L_{b}\right)$ state. If the estimates of the position of $S_{2,0}$ are cor- rect $\left(\approx 28000 \mathrm{~cm}^{-1}[57]\right)$, in principle $S_{2}$ can be populated by TTA. However, since $S_{2,0}$ has u symmetry, on the basis of the symmetry considerations in section 2.2 no preferential primary population of $\mathrm{S}_{2}$ can be expected.

\subsection{Delayed luminescence from 9-methylanthracene}

We measured the delayed-luminescence spectrum and the absorption spectrum of 9-methylanthracene in isopentane/cyclopentane (see fig. 10). The quantum yield of intersystem crossing $S_{1} \leadsto T_{1}$ of 9-methylanthracene strongly decreases with decreasing temperature $[58,59]$, and the same is true for the intensity of the delayed fluorescence at constant excitation power under the conditions of dominant triplet decay due to TTA. We were not able to measure the spectrum of the delayed luminescence up to $2 E\left(\mathrm{~T}_{1,0}\right)$ at $110 \mathrm{~K}$; for this reason we have measured the delayed-luminescence spectrum at $145 \mathrm{~K}$. The absorption spectrum was measured at $110 \mathrm{~K}$. Since the $S_{1}$ absorption band of 9-methylanthracene slightly shifts to lower energy with decreasing temperature,

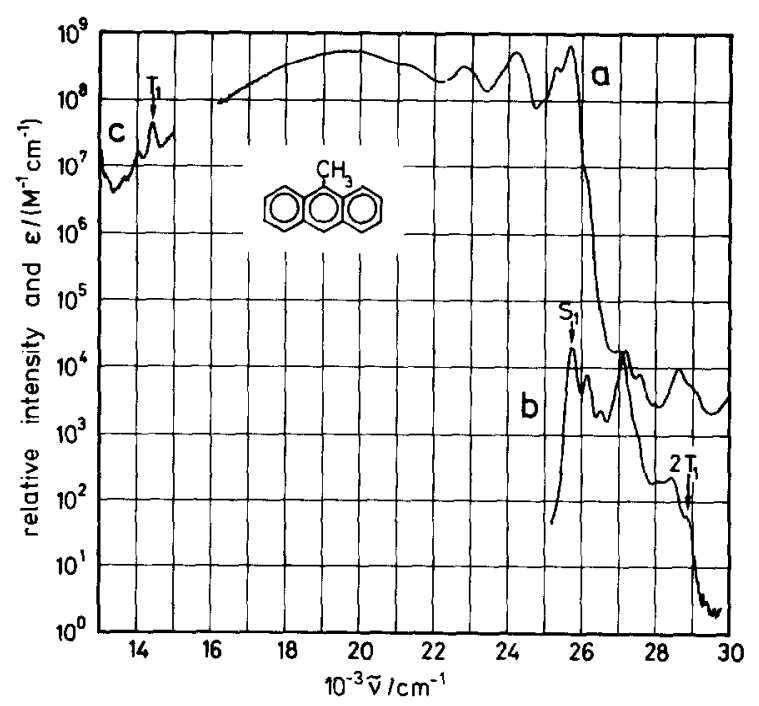

Fig. 10. (a) Spectrally corrected spectrum of the delayed luminescence from a solution of $5.0 \times 10^{-5} \mathrm{M}$ 9-methylanthracene in isopentane/cyclopentane $(7: 3)$ at $145 \mathrm{~K}$ and (b) absorption spectrum $\epsilon(\tilde{\nu})$ of 9-methylanthracene at $110 \mathrm{~K}$. 9-methylanthracene was excited with all lines of the argon ion laser in the near UV $(364,351$ and $333 \mathrm{~nm})$. The uncorrected phosphorescence spectrum (c) was measured at $106 \mathrm{~K}$. 
the energy differences between corresponding vibronic bands in absorption and in hot-band delayed fluorescence are a little too small.

The two spectra (a) and (b) in fig. 10 are very similar to the corresponding spectra of anthracene in fig. 9 . In particular the inhomogeneously broadened absorption spectra have practically the same structure, and the same classification and assignment of vibronic contour bands in the spectral range of hotband delayed fluorescence can be applied. Differences in the hot bands 1 to 3 are mainly due to a higher relative intensity of equilibrium hot-band delayed fluorescence in the case of 9-methylanthracene.

9-methylanthracene has no center of symmetry. Hence in principle the symmetry arguments of section 2.2 can no longer be applied. Nevertheless the relative intensities of the bands no. 8 and no. 9 (cf. table 2) are very similar to those of anthracene. If we assume that the symmetry considerations of section 2.2 are basically correct, then the similarity of the spectra in figs. 9 and 10 can mean, that the methyl group in 9-methylanthracene represents only a small perturbation of the $D_{2 h}$ symmetry of anthracene, and that the symmetry arguments are still applicable.

\subsection{Delayed luminescence from 1,2-benzanthracene}

1,2-benzanthracene (1,2-BA) has been the first compound with which a delayed fluorescence from upper excited singlet states has been observed $[2,60]$. We measured the delayed-luminescence spectrum and the absorption spectrum of 1,2-BA in isopentane/cyclopentane at 110 and $193 \mathrm{~K}$ (see fig. 11 ). We use the same phenomenological numbering of excited states as in refs. $[2,60]$. The following spectral ranges can be distinguished in the spectrum of the delayed luminescence at $110 \mathrm{~K}$ :

(a) $\tilde{y} \$ 17000 \mathrm{~cm}^{-1}$ : structured phosphorescence with a background of delayed excimer fluorescence;

(b) $17000 \mathrm{~cm}^{-1} \leqslant \tilde{\nu} \leqslant 20500 \mathrm{~cm}^{-1}$ : dominant structureless delayed excimer fluorescence;

(c) $20500 \mathrm{~cm}^{-1} \leqslant \tilde{\nu} \leqslant 26200 \mathrm{~cm}^{-1}$ : structured delayed $S_{1} \rightarrow S_{0}$ monomer fluorescence;

(d) $26200 \mathrm{~cm}^{-1} \leqslant \tilde{\nu} \leqslant 27000 \mathrm{~cm}^{-1}$ : hot bands of delayed $\mathrm{S}_{1} \rightarrow \mathrm{S}_{0}$ fluorescence;

(e) $27000 \mathrm{~cm}^{-1} \leqslant \tilde{\nu} \leqslant 29500 \mathrm{~cm}^{-1}$ : dominant delayed monomer-like $S_{2} \rightarrow S_{0}$ fluorescence;

(f) $29500 \mathrm{~cm}^{-1} \leqslant \tilde{\nu} \leqslant 33500 \mathrm{~cm}^{-1}$ : dominant delayed monomer-like $S_{3} \rightarrow S_{0}$ fluorescence;

(g) $33500 \mathrm{~cm}^{-1} \leqslant \tilde{\nu} \leqslant 34400 \mathrm{~cm}^{-1}$ : an extremely weak and temperature-dependent delayed luminescence extending $\approx 700 \mathrm{~cm}^{-1}$ above $2 E\left(T_{1,0}\right)$.

In the following discussion of the delayed luminescence we treat first the spectral ranges (b) to (f) and then the range $(\mathrm{g})$.

\subsubsection{Delayed fluorescence from 1,2-benzanthracene \\ The decrease of the relative intensity $I_{\mathrm{DFE}} / I_{\mathrm{DF} 1}$ of the delayed excimer fluorescence with decreasing temperature in range (b) is qualitatively the same as in the case of pyrene (cf. section 5.1.3). The stronger reduction of $I_{\mathrm{DFE}} / I_{\mathrm{DFI}}$ in the case of $1,2-\mathrm{BA}$ is prob-}

Table 2

Assignment of vibronic contour bands in the spectrum of the hot-band delayed luminescence from 9-methylanthracene to vibronic contour bands in the absorption spectrum of 9-methylanthracene - see fig. 10. Abbreviations: m: maximum, s: shoulder, ws: weak shoulder

\begin{tabular}{|c|c|c|c|c|c|}
\hline No. & $\begin{array}{l}\text { Del. lumin. } \\
\left(\mathrm{cm}^{-1}\right)\end{array}$ & Type & Intensity & $\begin{array}{l}\text { Absorption } \\
\left(\mathrm{cm}^{-1}\right)\end{array}$ & Type \\
\hline 0 & 25660 & $\mathbf{m}$ & 1 & 25740 & $\mathrm{~m}$ \\
\hline 1 & 26050 & $\mathbf{s}$ & $1.4 \times 10^{-2}$ & 26155 & $\mathrm{~m}$ \\
\hline 2 & 26370 & ws & $3.2 \times 10^{-4}$ & 26510 & $\mathbf{m}$ \\
\hline 3 & - & - & - & 26935 & $\mathbf{s}$ \\
\hline 4 & 26965 & $\mathbf{m}$ & $2.6 \times 10^{-5}$ & 27170 & $\mathbf{m}$ \\
\hline 5 & 27465 & s & $1.8 \times 10^{-6}$ & 27580 & m. \\
\hline 6 & 27740 & ws & $3.6 \times 10^{-7}$ & 27965 & $\mathbf{m}$ \\
\hline 7 & 28065 & m & $2.8 \times 10^{-7}$ & 28320 & $s$ \\
\hline 8 & 28415 & $\mathbf{m}$ & $3.2 \times 10^{-7}$ & 28610 & $\mathbf{m}$ \\
\hline 9 & 28790 & $s$ & $8.5 \times 10^{-8}$ & 29000 & s \\
\hline $2 T_{1,0}$ & 28840 & & & & \\
\hline
\end{tabular}




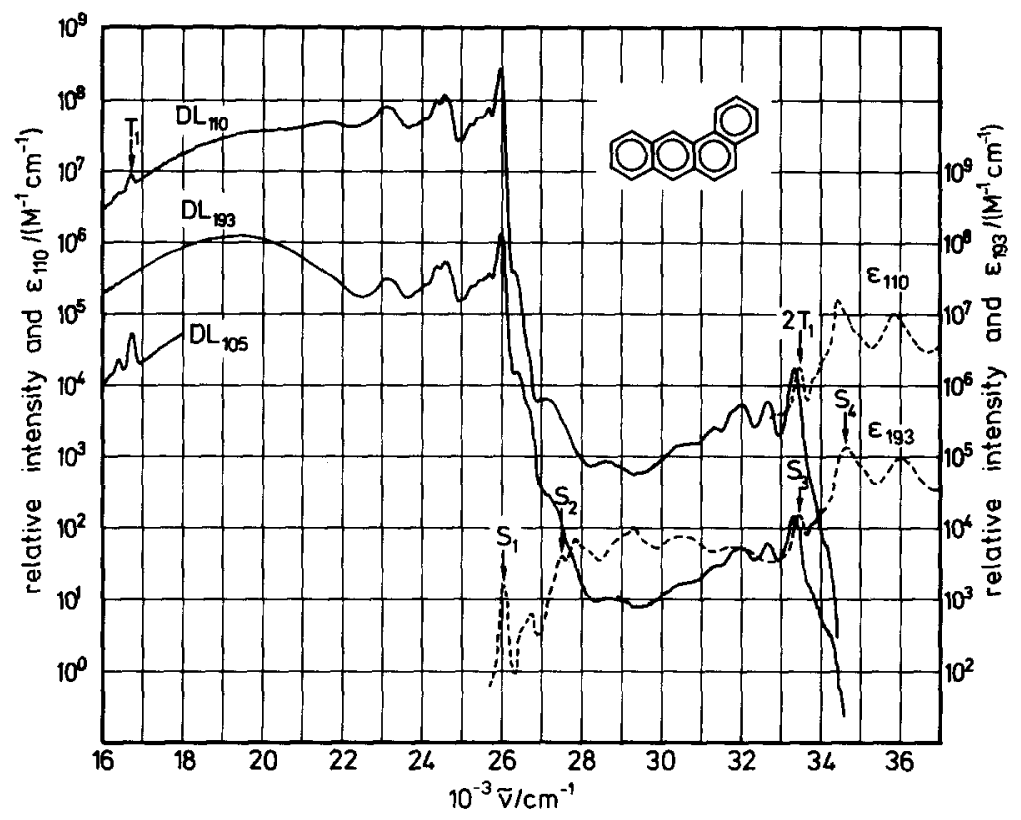

Fig. 11. Spectrally corrected delayed-luminescence spectra (-) of a $1.0 \times 10^{-5} \mathrm{M}$ solution of 1,2-benzanthracene in isopentane/ cyclopentane $(7: 3)$ and absorption spectra $\epsilon(\tilde{\nu})(-)$ at 110 and $193 \mathrm{~K}$ each. The excitation wavelength was $363.8 \mathrm{~nm}$. The spectrum $\mathrm{DL}_{105}$ has been measured with a different photomultiplier and has not been corrected.

ably connected with the shorter lifetime of $S_{1}$ of 1,2BA. Labhart and Wyrsch [27] studied the temperature dependence of $I_{\mathrm{DFE}} / I_{\mathrm{DF} 1}$ of 1,2-BA in ethanol and in a mixture of ethanol and ethylene glycol, and they developed a quantitative model for its description.

The assignment of range (d) to hot bands of delayed $S_{1} \rightarrow S_{0}$ fluorescence is based on the strong temperature dependence of the relative intensity of range (d). In range (e) at $110 \mathrm{~K}$, non-equilibrium hot-band delayed fluorescence from $S_{1}$ and delayed fluorescence from $S_{2}$ are dominant; at the $S_{2}$ origin in absorption $\left(27500 \mathrm{~cm}^{-1}\right)$, a clear distinction between both kinds of delayed fluorescence is not possible. At $193 \mathrm{~K}$, equilibrium hot-band delayed fluorescence is dominant up to $\approx 27500 \mathrm{~cm}^{-1}$ (possibly, part of this hot-band delayed fluorescence is delayed $S_{2} \rightarrow S_{0}$ fluorescence - cf. ref. [61 ]).

In the spectral range (f), the only significant difference between the two spectra is a slightly smaller width of the vibronic contour bands at the lower temperature. In the following the quantum yield $\phi_{\mathrm{DF} 3}$ of delayed $S_{3} \rightarrow S_{0}$ fluorescence will be of interest. We define the quantum yield $\phi_{\mathrm{DF} n}$ of the delayed fluores- cence from $S_{n}$ by the equation

$$
\phi_{\mathrm{DF} n}=I_{\mathrm{DF} n} /\left(I_{\mathrm{DF} 1} / \phi_{\mathrm{F} 1}+I_{\mathrm{DFE}} / \phi_{\mathrm{FE}}\right)
$$

With eq. (23), the data of ref. [2], and $\phi_{\mathrm{FE}}=0.47$, and $\phi_{\mathrm{F} 1}=0.19$ at room temperature (table 7.3 of ref. [36]), we obtain

$\phi_{\mathrm{DF} 3}=1.1 \times 10^{-5}$.

One may ask whether the delayed luminescence in the spectral range (f) can be interpreted as radiative TTA. Though the corrected phosphorescence spectrum of 1,2-BA is not available to us, also from uncorrected phosphorescence spectra $[26,60]$, it is obvious that the calculated spectrum of radiative TTA would look completely different from the observed spectrum of the delayed luminescence in range $(f)$. An additional argument for the interpretation of range (f) as delayed fluorescence comes from a quantum mechanical calculation of the spectrum of the $S_{3} \rightarrow S_{0}$ fluorescence from 1, 2-BA by Gustav and Seydenschwanz [62], who calculated a vibrational structure 
and intensity distribution similar to the observed spectrum in range ( $f$ ).

\subsubsection{Delayed luminescence from}

1.2-benzanthracene above $2 E\left(T_{i, a}\right)$

The extension of the spectrum of the delayed luminescence from a solution of 1,2-BA beyond $2 E\left(T_{1,0}\right)$ has been puzzling. Different assignments of range (g) are conceivable:

(I) Delayed $S_{4} \rightarrow S_{0}$ and nonequilibrium hot-band delayed $S_{3} \rightarrow S_{0}$ fluorescence due to nonradiative TTA of vibrationally excited triplets:

$$
\begin{array}{r}
\mathbf{T}_{1, v 1}+\mathbf{T}_{1, v 2} \rightsquigarrow \mathrm{S}_{4,0}+\mathrm{S}_{0,0} \\
\rightsquigarrow \mathrm{S}_{3, v}+\mathrm{S}_{0,0},
\end{array}
$$

with $\tilde{\nu}_{v_{1}}+\tilde{\nu}_{v_{2}} \gtrsim 1000 \mathrm{~cm}^{-1}$ in (25a).

(II) Radiative TTA of vibrationally excited triplets

$\mathrm{T}_{1, v 1}+\mathrm{T}_{1, v 2} \rightarrow \mathrm{S}_{0,0}+\mathrm{S}_{0,0}+\mathrm{h} \nu_{\mathrm{rad}}$.

(III) Other interpretations of range (g).

Independently of the interpretation (I) or (II), an interpretation in terms of annihilation of vibrationally excited triplets should not be considered as implausible on the following grounds. Firstly, according to the considerations in section 2 , hot bands in the phosphorescence should automatically lead to radiative TTA extending beyond $2 E\left(T_{1,0}\right)$ (cf. eq. (7)) and to a population of vibronic states $S_{n, v}$ above $2 E\left(T_{1,0}\right)$ by nonradiative TTA (cf. section 2.2 ). Secondly, as expected for a thermally activated process, the relative intensity $I_{\mathrm{DL}}(\mathrm{g}) / I_{\mathrm{DF} 3}$ of range $(\mathrm{g})$ increases with increasing temperature. Thirdly, as already mentioned in the introduction, with azulene a "thermally activated" delayed $S_{2} \rightarrow S_{0}$ fluorescence can be observed [15], which corresponds to $\tilde{\nu}_{v 1}+$ $\tilde{v}_{v z} \approx 600 \mathrm{~cm}^{-1}$. Thus the interpretation (I) is not unprecedented.

(I) Interpretation of range $(g)$ as delayed fluorescence

For the interpretation of part of the delayed luminescence in range $(\mathrm{g})$ as delayed $S_{4} \rightarrow S_{0}$ fluorescence, an estimate of the quantum yield $\phi_{\mathrm{F} 4}$ of the prompt $\mathrm{S}_{4} \rightarrow \mathrm{S}_{0}$ fluorescence from 1,2-BA is needed. A fluorescence from upper excited singlet states of 1,2-BA has been observed with consecutive two-photon excitation [63-68];
$\mathrm{S}_{0} \stackrel{\ddot{D}_{1}}{\longrightarrow} \mathrm{S}_{2} \stackrel{+}{ } \mathrm{S}_{1} \stackrel{\ddot{\nu}_{2}}{\longrightarrow} \mathrm{S}_{n}$.

The observed upper-state fluorescence was interpreted as dominant $S_{4} \rightarrow S_{0}$ fluorescence. This interpretation is questionable for several reasons. Firstly, the strongest band of the $S_{4} \rightarrow S_{0}$ fluorescence should be only slightly red-shifted $\left(<500 \mathrm{~cm}^{-1}\right)$ relative to the 0,0 transition of the $S_{0} \rightarrow S_{4}$ absorption band. The observed maximum [63-66] is, however, in the spectral range of the $S_{3} \rightarrow S_{0}$ fluorescence. According to ref. [67], the quantum yield of the $S_{4} \rightarrow S_{0}$ fluorescence is practically independent of the wavenumber $\tilde{\nu}_{2}$ of the second photon: $\phi_{\mathrm{F} 4}=(2.0 \pm 0.5) \times 10^{-5}$. This value is only slightly greater than $\phi_{\mathrm{DF} 3}=1.1 \times 10^{-5}$ (cf. eq. (24)). The relation between the quantum yields $\phi_{\mathrm{F} 3}$ of the prompt fluorescence and $\phi_{\mathrm{DF} 3}$ of the delayed fluorescence from $S_{3}$ is $\phi_{\mathrm{F} 3}=\phi_{\mathrm{DF} 3} / p_{3}$, where $p_{3} \leqslant 1$ is the probability of formation of $S_{3}$ in the singlet channel of TTA; thus we have $\phi_{\mathrm{F} 4} \leqslant 2 \phi_{\mathrm{F} 3}$. If we postulate as in ref. [67] the validity of the cascade model of electronic relaxation and assume that internal conversion $S_{4} \leadsto S_{3}$ takes place with unit probability, one may assume that a large fraction of the observed upper-state fluorescence in refs. $[63-68]$ has to be assigned to $S_{3} \rightarrow S_{0}$ fluorescence and not to $S_{4} \rightarrow S_{0}$ fluorescence.

The fact that makes the assignment of range $(\mathrm{g})$ to delayed $S_{4} \rightarrow S_{0}$ fluorescence doubtful are the quantitative aspects of $I_{\mathrm{DL}}(\mathrm{g}) / I_{\mathrm{DF} 3}$. Let $k_{\mathrm{nri}}$ be the rate constant for the primary generation of $S_{i}$ by nonradiative TTA in an encounter complex of two molecules in $\mathrm{T}_{1}$. We tentatively assume $k_{\mathrm{nr} 3}$ as temperature independent and $k_{\mathrm{nr} 4}=k_{\mathrm{nr}}^{(0)} \exp \left(-\Delta E / k_{\mathrm{B}} T\right)$, where $\Delta E=E\left(\mathrm{~S}_{4,0}\right)-2 E\left(\mathrm{~T}_{1,0}\right) \approx h c \times 1000 \mathrm{~cm}^{-1}$. The observed value of $I_{\mathrm{DL}}(\mathrm{g}) / I_{\mathrm{DF} 3}$ would imply $k_{\mathrm{nr} 4}^{(0)} \gg$ $k_{\mathrm{nr} 3}$; if nonradiative TTA is treated as a special case of Dexter energy transfer as in section $2.2, k_{\mathrm{nr}}^{(0)} \gg$ $k_{\mathrm{nr} 3}$ would mean that the transition $\mathrm{T}_{1} \rightarrow \mathrm{S}_{4}$ is much stronger than the transition $T_{1} \rightarrow S_{3}$. The temperature dependence of $I_{\mathrm{DL}}(\mathrm{g}) / I_{\mathrm{DF} 3}$ is, however, significantly weaker than would correspond to the value of $\Delta E$. Therefore, as far as the delayed luminescence in range (g) can be interpreted as delayed fluorescence, most of this delayed fluorescence is likely to be nonequilibrium hot-band delayed fluorescence from $\mathrm{S}_{3}$ due to nonradiative TTA (25b).

(II) Interpretation of range $(g)$ as radiative TTA 
The dominant terms of the theoretical expression for the rate constant $k_{\mathrm{r}}$ of radiative TTA [5] are proportional to the oscillator strengths $f_{0 n}$ of allowed transitions $S_{0} \rightarrow S_{n}$ and inversely proportional to $\left|\Delta E_{n}\right|^{2}=\left|E\left(\mathrm{~S}_{n}\right)-2 E\left(\mathrm{~T}_{1}\right)\right|^{2}$ (if a damping term is neglected):

$k_{\mathrm{r}} \propto \sum_{n} f_{0 n} /\left|E\left(\mathrm{~S}_{n}\right)-2 E\left(\mathrm{~T}_{1}\right)\right|^{2}$.

With respect to eq. (27), 1,2-BA is a promising compound for radiative TTA: The transition $\mathrm{S}_{0} \rightarrow \mathrm{S}_{4}$ is very strong and $\Delta E_{4}$ is small. A quantitative estimate of the temperature dependence of the relative intensity $I_{\mathrm{rad}} / I_{\mathrm{DF} 3}$ of radiative TTA is difficult on several grounds. In the case of the radiative annihilation (26) of vibrationally excited triplets, the spectrum of radiative TTA is given by

$I_{\mathrm{rad}}(\tilde{\nu})=C \sum_{v_{1}, v_{2}} p_{\nu_{1}, v_{2}} k_{r}\left(v_{1}, v_{2}\right) I_{\mathrm{rad}}\left(\tilde{\nu}, v_{1}, v_{2}\right)$,

where $C$ is a constant, $p_{v_{1}, v_{2}}$ is the relative probability for a vibrationally excited triplet pair $T_{1, v_{1}} \ldots T_{1, v_{2}}$, $k_{\mathrm{r}}\left(v_{1}, v_{2}\right)$ is the rate constant for radiative TTA of this pair, and $I_{\mathrm{rad}}\left(\tilde{\nu}, v_{1}, v_{2}\right)$ is the corresponding spectrum of radiative TTA.

For the vibrational excitation of molecules in the triplet state one may assume thermal equilibrium: $p_{v_{1}, v_{2}} \propto \exp \left[-\left(E_{v_{1}}+E_{v_{2}}\right) / k_{\mathrm{B}} T\right]$. For $k_{\mathrm{r}}=k_{\mathrm{r}}\left(v_{1}, v_{2}\right)$, eq. (27) has to be modified as follows:

$$
k_{\mathrm{r}}\left(v_{1}, v_{2}\right) \propto f_{0 n} /\left|E\left(\mathrm{~S}_{n, 0}\right)-E\left(\mathrm{~T}_{1, v_{1}}\right)-E\left(\mathrm{~T}_{1, v_{2}}\right)\right|^{2} .
$$

$I_{\text {rad }}\left(\tilde{\nu}, v_{1}, v_{2}\right)$ can be calculated in principle in the same way as $I_{\text {rad }}(\tilde{v})$ in eq. (11), if the spectra of the phosphorescence from $T_{1, v_{1}}$ and $T_{1, v_{2}}$ are known. The integrand of the integral in eq. (7) is to be replaced by $F_{\mathrm{P}, \nu_{1}}\left(\tilde{\nu}^{\prime}\right) F_{\mathrm{P}, \nu_{2}}\left(\tilde{\nu}-\tilde{\nu}^{\prime}\right)$. The comparison of eqs. (28) and (29) shows that the decrease of $p_{v_{1}, \nu_{2}}$ with increasing vibrational energy is partly compensated by a simultaneous increase of $k_{\mathrm{r}}\left(v_{1}, v_{2}\right)$. Hence the hot delayed luminescence due to radiative TTA may exhibit a decrease with increasing wavenumber that is slower than the typical decrease of an equilibrium hot fluorescence at the same temperature. In this respect, the observed intensity distribution in range $(\mathrm{g})$ would be an argument in favour of radiative TTA.

(III) Other interpretations of range $(g)$

One may ask whether the origin of the phosphores- cence from 1,2-BA is a false origin. A false origin would mean that the true triplet energy is higher than assumed. There are examples of compounds with a false origin of the phosphorescence (e.g. biphenylene [25]). However, we found in the literature no hint to a false origin of the phosphorescence from 1,2-BA, and because of the low symmetry of 1,2-BA a false origin is not likely.

An impurity (') of type (d) (cf. section 4) with $E\left(\mathrm{~T}_{1}^{\prime}\right)>E\left(\mathrm{~T}_{1}\right)$ and $E\left(\mathrm{~S}_{1}^{\prime}\right)>E\left(\mathrm{~S}_{1}\right)$ could contribute to the delayed luminescence in range $(\mathrm{g})$ as delayed fluorescence or radiative TTA due to homo-TTA of $2 T_{1}^{\prime}$ or hetero-TTA of $T_{1}^{\prime}$ and $T_{1}$. We have seen no convincing way to interpret the delayed luminescence in range $(\mathrm{g})$ as an impurity effect

(1) We first consider the possibility that the delayed luminescence in range $(\mathrm{g})$ is due to nonradiative or radiative hetero-TTA. If we assume an equilibrium for the relative population of the triplet states $T_{1}^{\prime}$ and $T_{1}$, then for the corresponding concentrations we have the ratio $c_{\mathrm{T}}^{\prime} / c_{\mathrm{T}}=c_{0}^{\prime} / c_{0} \times \exp \left(-\Delta E / k_{\mathrm{B}} T\right)$, where $c_{0}^{\prime}$ and $c_{0}$ are ground-state concentrations and $\Delta E=E\left(\mathrm{~T}_{1}^{\prime}\right)-E\left(\mathrm{~T}_{1}\right)$. Since $c_{0}^{\prime} / c_{0} \ll 1$, the reaction (25) with $E_{\nu_{1}}+E_{\nu_{2}}=\Delta E$ is much more likely than the hetero-annihilation of $T_{1}^{\prime}$ and $T_{1}\left(p_{\nu_{1}, v_{2}} \gg p_{0,0^{\prime}}\right)$. The same is true for radiative TTA (26).

(2) A higher relative concentration of $T_{1}^{\prime}$ than the equilibrium concentration can be reached by direct excitation of an impurity. However, since this is possible only if $\tilde{\nu}_{0,0}\left(S_{0}^{\prime} \rightarrow S_{1}^{\prime}\right)$ is smaller than or equal to the excitation wavenumber $\tilde{y}_{\text {exc }} \approx 27500 \mathrm{~cm}^{-1}$, the delayed luminescence in range $(\mathrm{g})$ could be at most a very weak delayed fluorescence from an upper excited singlet state. Moreover, due to the diffusioncontrolled triplet energy transfer $T_{1}^{\prime}+S_{0} \leadsto S_{0}^{\prime}+T_{1}$, the relative intensity of range $(\mathrm{g})$ would depend on the viscosity - in contrast to the experimental result (at $193 \mathrm{~K}$, the relative intensity of range $(\mathrm{g})$ is with 1,2-BA in isopentane/cyclopentane (fig. 11) the same as with $1,2-\mathrm{Ba}$ in methylcyclohexane [2]).

\subsection{Radiative and nonradiative TTA - general discussion}

On the basis of the five investigated compounds and of the proposed procedure for the calculation of the spectrum of radiative TTA, the following combination of properties is favourable for the observation 
of radiative TTA and its distinction from delayed fluorescence:

(1) The molecules have a center of symmetry.

(2) The lowest triplet state has u symmetry.

(3) In the neighborhood of $2 E\left(T_{1,0}\right)$ the molar extinction coefficient is only moderately large $\left(<10^{4}\right.$ $\mathrm{M}^{-1} \mathrm{~cm}^{-1}$ ) and decreases with increasing wavenumber.

(4) $2 E\left(T_{1,0}\right)$ is in a range of strong intermediate vibronic coupling of excited singlet states.

(5) The lifetimes of vibronic singlet states in the neighborhood of $2 E\left(\mathrm{~T}_{1,0}\right)$ are very short.

(6) A singlet $\mathrm{g}$ state is accessible by nonradiative TTA.

Of the five investigated compounds, pyrene and phenazine represent this combination of properties. Another compound with the same combination of properties and a spectrum of the delayed luminescence extending up to $2 E\left(T_{1,0}\right)$ is naphthalene [69]. The interpretation of the high-energy part of an observed delayed luminescence as radiative TTA has been based on a positive argument, the similarity of the observed spectrum with a calculated spectrum, and on the negative argument that the delayed luminescence at $2 E\left(T_{1,0}\right)$ cannot be delayed fluorescence. We discuss the validity of both arguments in turn.

\subsubsection{On the predictability of the spectrum of radiative TTA}

The derivation of formula (11) for the spectrum of radiative TTA may seem plausible but is of course not a derivation in a theoretical sense. In the case of the bimolecular radiative annihilation of metastable singlet oxygen, eq. (11) does not yield the correct intensity distribution of the dimol luminescence [11]. The intensity ratio of the 0,0 and 0,1 transitions of the $\mathrm{O}_{2}\left({ }^{1} \Delta_{\mathrm{B}}\right) \rightarrow \mathrm{O}_{2}\left({ }^{3} \Sigma_{\mathrm{g}}^{-}\right)$phosphorescence is $I_{0,0} /$ $I_{0,1} \approx 70[70,71]$. According to eq. (7), from this value the intensity ratio $r=$ $I_{\text {rad }}^{*}(0,0 ; 0,0) / I_{\text {rad }}^{*}(0,0 ; 0,1) \approx 30$ would follow. The experimental value of $r$, however, is always $0.5 \$ r \$ 1.0$ under very different experimental conditions [11,72-76]. An example closer to the present investigation is an absorption band of naphthalene $(\mathrm{N})$ in the presence of oxygen, which can be assigned to the cooperative transition [77]:

\footnotetext{
${ }^{3}\left[\mathrm{~N}\left(\mathrm{~S}_{0}\right) \ldots \mathrm{O}_{2}\left({ }^{3} \Sigma_{\mathrm{g}}^{-}\right)\right] \rightarrow{ }^{3}\left[\mathrm{~N}\left(\mathrm{~T}_{1}\right) \ldots \mathrm{O}_{2}\left({ }^{1} \Delta_{\mathrm{g}}\right)\right]$.
}

Unfortunately, due to a background of oxygen-induced transitions to higher triplet states, a reliable determination of the spectrum of this cooperative transition is not possible.

In special cases eq. (7) could be justified by the quantum mechanical calculation of the spectra of phosphorescence and radiative TTA and by a good agreement of the theoretical spectrum of radiative TTA with the spectrum of radiative TTA calculated with eq. (7) from the theoretical phosphorescence spectrum. As a first example, naphthalene would be the compound of most interest. In this connection we mention that, due to the high solubility of naphthalene it would be possible to measure the complete spectrum of the delayed luminescence from naphthalene down to $77 \mathrm{~K}$ by using fluid mixtures of propane, isobutane and butane as solvent. At $77 \mathrm{~K}$ the assumption that all triplet molecules are in $T_{1,0}$ would be justified to a high degree.

Our model implies the assumption that the relative intensity of a vibronic phosphorescence band is not changed by triplet-triplet interaction. The justification of this assumption can be discussed from an empirical point of view. Spin-forbidden transitions are known to be strongly enhanced by heavy atoms [78] or by oxygen [79] (for a systematic treatment of these effects and a review of the literature cf. ref. [80]). Though the probability of a spin-forbidden transition can be enhanced by several orders of magnitude by these effects, the spectrum of the transition remains essentially unchanged. Hence one may expect that the effect of triplet-triplet interaction on the spectrum of a transition in a single molecule is also small. In principle, the effect of triplet-triplet interaction on the phosphorescence spectrum is observable, since at high triplet concentrations there must be an enhancement of phosphorescence due to triplettriplet interaction. In practice, the detection of this enhancement would be very difficult due to the complications arising from TTA.

\subsubsection{On the interpretation of nonradiative TTA as a case of Dexter energy transfer}

If nonradiative TTA is interpreted as a case of Dexter energy transfer, one meets the following difficulty: The electronic transition in the energy-accepting molecule is spin-forbidden in the singlet channel $\left(T_{1} \rightarrow \mathrm{S}_{n}\right)$ and spin-allowed in the triplet 
channel $\left(T_{1} \rightarrow T_{m}\right)$. Hence one might expect for the corresponding probabilities $p_{\mathrm{S}}$ and $p_{\mathrm{T}}$ of both channels the relation $p_{\mathrm{S}} \ll p_{\mathrm{T}}$. Experimental values of $p_{\mathrm{S}}$ are, however, often close to the spin-statistical value $1 / 4[1,81-85]$ (the lowest quintet state can be assumed to be not accessible by TTA $[4,86]$ ). In the following we show that $p_{\mathrm{S}} \approx 1 / 4$ is no argument against the interpretation of nonradiative TTA as a case of Dexter energy transfer.

An independence of $p_{\mathrm{S}}$ on the specific properties of the electronic states of a compound can be rationalized by a two-step mechanism of nonradiative TTA. The first and rate-determining step is the diffusioncontrolled formation of a triplet pair ${ }^{i}\left(T_{1} \ldots T_{1}\right)$ with definite total spin, characterized by the multiplicity $i=1,3,5$. The second step is the $i$-specific TTA, characterized by the first-order rate constant ${ }^{i} k_{\mathrm{nr}}\left({ }^{\prime} k_{\mathrm{nr}}\right.$ is identical with the rate constant $k_{\mathrm{nr}}$ in previous sections). By neglecting reencouter effects, the secondorder rate constant ${ }^{i} k_{\mathrm{TTA}}$ of the $i$-channel of TTA can be written in the form

${ }^{i} k_{\mathrm{TTA}}={ }^{i} p k_{\text {enc }} \frac{{ }^{i} k_{\mathrm{nr}}}{k_{\mathrm{nr}}+k_{\mathrm{diss}}}$,

where ${ }^{i} p=i / 9, k_{\text {enc }}$ is the second-order rate constant of diffusion-controlled formation of triplet encounter complexes, and $k_{\text {diss }}$ is the rate constant of encounter complex dissociation. According to eq. (30) the spin-statistical value $p_{\mathrm{S}}=1 / 4$ results from a nonreactive quintet channel $[4]\left({ }^{5} k_{\mathrm{nr}}=0\right)$ and from the relation ${ }^{i} k_{\mathrm{nr}} \gg k_{\text {diss }}(i=1,3): p_{\mathrm{s}}={ }^{1} p /\left({ }^{1} p+{ }^{3} p\right)$.

The interpretation of nonradiative TTA as Dexter energy transfer implies the relation ${ }^{1} k_{\mathrm{nr}}<{ }^{3} k_{\mathrm{nr}}$ or $p_{\mathrm{S}}<1 / 4$. The strongest argument against this interpretation would be an example of $p_{\mathrm{S}}>1 / 4$. Such examples have been reported in the literature (for a compilation of $p_{\mathrm{S}}$ values $\mathrm{cf}$. ref. [82]). However, since the experimental determination of $p_{\mathrm{s}}$ is difficult, small positive deviations from $p_{S}=1 / 4$ are not necessarily significant. On the other hand, there are many examples for $p_{\mathrm{s}}$ being significantly or even much smaller than $1 / 4[81,82]$. Within the Dexter model of TTA, two different explanations of $p_{\mathrm{S}}<1 / 4 \mathrm{can}$ be given. (I) On the basis of eq. (30), $p_{\mathrm{S}}<1 / 4$ would be a consequence of ${ }^{3} k_{\mathrm{nr}} \gg k_{\text {diss }}$ and ${ }^{1} k_{\mathrm{nr}} \lesssim k_{\text {diss. }}$ (II) Eq. (30) is based on the two assumptions that at all intermolecular distances (1) the formation of a triplet pair with definite total spin is much faster than TTA and
(2) relaxation of the total spin in an encounter complex can be neglected; both assumptions can be wrong. Explanation (I) may apply to centrosymmetric molecules like anthracene $\left(p_{\mathrm{S}} \approx 0.08[82]\right)$ or perylene $\left(p_{\mathrm{S}} \approx 0.02\right.$ [81]) with no $\mathrm{g}$ state accessible by TTA. If this explanation is correct, $p_{\mathrm{S}}$ should increase with increasing solvent viscosity. Investigations of the dependence of $p_{\mathrm{S}}$ on solvent viscosity and temperature are not known to us. Explanation (II) may apply in cases where ${ }^{3} k_{\mathrm{nr}}$ is unusually large and spin-lattice relaxation in the individual triplet state is very fast. A conceivable example for explanation (II) is phenanthrene $\left(p_{\mathrm{S}} \approx 0.02[81,83]\right)$. An unusually large value of ${ }^{3} k_{\mathrm{nr}}$ can be expected for phenanthrene, because its phosphorescence spectrum overlaps with a strong band of the triplet-triplet absorption spectrum [43]. Spin-lattice relaxation in the triplet state of phenanthrene is unusually fast even at very low temperatures [ 87 ], and the rate constant of the total triplet decay due to TTA is only weakly magnetic-field dependent [88].

In conclusion, there are indications of the importance of the overlap integral of the phosphorescence spectrum with the $T_{1} \rightarrow S_{n}$ or $T_{1} \rightarrow T_{m}$ absorption spectrum in nonradiative TTA. Again, as in the case of radiative TTA, one may ask to what extent triplettriplet interaction may change the phosphorescence spectrum and the $T_{1} \rightarrow S_{n}$ absorption spectrum. The point of interest here is whether in the case of centrosymmetric molecules the parity selection rule still applies in the presence of triplet-triplet interaction. An argument in favour therefor is the oxygen-induced $S_{0} \rightarrow T_{1}$ absorption band of biphenylene [89]: The parity forbiddance of the 0,0 transition [25] is not relaxed by the interaction with oxygen. Thus, inasmuch as the optical transition $T_{1} \rightarrow S_{n}$ is important in the singlet channel of nonradiative TTA, the assignment of a low-temperature delayed luminescence at $2 E\left(T_{1,0}\right)$ to radiative TTA is reasonable in the case of centrosymmetric molecules.

\subsection{On the indirect determination of lifetimes of upper excited singlet states}

The lifetime of an upper excited singlet state $S_{n}$ ( $n>1$ ) can be calculated from the quantum yield $\phi_{\mathrm{DF} n}$ of the delayed $S_{n} \rightarrow S_{0}$ fluorescence, if three assumptions are made [2]: 
(I) TTA leads with the probability $p_{n}=1$ to $S_{n}$, either directly or by internal conversion $S_{x} \rightsquigarrow S_{n}$ from a higher excited state $S_{x}$.

(II) Fluorescence $S_{n} \rightarrow S_{0}$ and internal conversion $S_{n} * S_{1}$ are the only decay processes of $S_{n}$. That is, due to the low value of $\phi_{\mathrm{DF} n}$, the relaxation of $S_{n}$ leads to $S_{1}$ with the probability $p_{1} \approx 1$ (Vavilov's rule).

(III) The radiative lifetime of $S_{n}$ can be calculated from the inhomogeneously broadened $S_{0} \rightarrow S_{n}$ absorption band.

With these assumptions, the lifetime $\tau_{n}^{\prime}$ of $\mathrm{S}_{n}$ is given by

$\tau_{n}^{\prime}=\tau_{\mathrm{F} n}^{*} \phi_{\mathrm{DF} n}$,

where $\tau_{\mathrm{F} n}^{*}$ is the radiative lifetime of $\mathrm{S}_{n}$ calculated with the Strickler-Berg formula [90] or with a similar formula (e.g. with eq. $\left(32,6^{\prime}\right)$ in ref. [16]). In the case of excimer-forming compounds, $\phi_{\mathrm{DF} n}$ is given by eq. (23).

In ref. [2] the assumptions (I)-(III) were considered as justified, and the lifetimes $\tau_{2}$ of $S_{2}$ pyrene and $\tau_{3}$ of $S_{3}$ of 1,2-BA were estimated with eq. (31). We now believe, that assumption ( $\mathrm{I}$ ) is doubtful and assumption (III) is definitely wrong in the case of $\mathrm{S}_{2}$ of pyrene. Assumption (I) is questionable, since $S_{3}\left(B_{1 g}\right)$ [18] is accessible by TTA, and a direct internal conversion $S_{3} \leadsto S_{1}$ is conceivable. Assumption (III) is wrong, because pyrene is an example of intermediate strong $S_{2}-S_{1}$ vibronic coupling $[22,23]$. Each zeroorder vibronic state $\mathrm{S}_{2, v}$ is split into a whole set of $\mathrm{S}_{2-}$ $S_{1}$ mixed vibronic states. As a consequence of this $S_{2^{-}}$ $S_{1}$ coupling, the average radiative lifetime of $S_{2}$ is much longer [19] than the radiative lifetime calculated with the Strickler-Berg formula [90] (for recent discussions of intermediate strong $S_{2}-S_{1}$ vibronic coupling cf. refs. $[20,21])$. In conclusion, in ref. [2] the estimated lifetime of $S_{2}$ of pyrene $(\approx 0.1$ ps) is too short by an order of magnitude or more.

If one gives up the assumptions (I)-(III), the appropriate formula for the true lifetime $\tau_{n}$ of $S_{n}$ is

$\tau_{n}=\left(N_{n} p_{1} / p_{n}\right) \tau_{\mathrm{F} n}^{*} \phi_{\mathrm{DF} n}=\left(N_{n} p_{1} / p_{n}\right) \tau_{n}^{\prime}$,

where $N_{n}$ accounts for the lengthening of the radiative lifetime resulting from strong intermediate vibronic coupling. $p_{1}=1$ corresponds to the validity of Vavilov's rule. Exceptions to Vavilov's rule are known (cf. section 5.14 in ref. [36]). The validity of Vavilov's rule can be checked by the measurement of the excitation spectrum of the prompt fluorescence from $S_{1}$. The comparison of eq. (32) with eq. (31) shows that, if Vavilov's rule applies, eq. (31) in general can yield only a lower bound for $\tau_{n \text { - }}$

For $S_{3}$ of 1,2-BA the situation is less clear than for $S_{2}$ of pyrene. With $\phi_{\mathrm{DF} 3}=1.1 \times 10^{-5}$ from eq. (24) and $\tau_{\mathrm{F} 3}^{*}=24$ ns [2], eq. (32) yields

$\tau_{3}=\left(N_{3} p_{1} / p_{3}\right) 0.26 \mathrm{ps}$.

Since in general deviations from Vavilov's rule are the less likely the larger a molecule is, we assume $p_{1} \approx 1$. A lower limit of $0.26 \mathrm{ps}^{\# 3}$ for the lifetime of $S_{3}$ represents already a rather long lifetime. Since on the one hand $p_{3}$ can be appreciably smaller than unity and on the other hand $\tau_{3} \lesssim 1 \mathrm{ps}$ is likely, $N_{3}$ is not likely to be greater than unity. $N_{3}=1$ corresponds to the statistical limit of $S_{3}-S_{2}$ vibronic coupling [19]. In the case of 1,2-BA, the low symmetry $\left(C_{s}\right)$ of this molecule is an argument in favour of the statistical limit. In a molecule with $C_{s}$ symmetry, in principle all in-plane vibrations can couple in-plane polarized electronic transitions; hence the density of vibronic states $S_{n-1, v}$, which can couple with $S_{n, 0}$, may be extremely high at lower energy gaps between $S_{n-1}$ and $S_{n}$ than in the case of molecules of the same size but with a high symmetry. If $S_{3}$ of 1, 2-BA is an example for the statistical limit, then the lifetime of $S_{3}$ can be determined indirectly by the measurement of the homogeneous width of the 0,0 transition of the $S_{0} \rightarrow S_{3}$ absorption band (with isolated molecules in a supersonic jet as in ref. [91] or in condensed phase with the method of transient spectral hole-burning $[92,93]$ ).

\section{Conclusion}

(1) The spectrum of radiative TTA can be calculated from the phosphorescence spectrum on the basis of three simple assumptions. The only two adjustable parameters are a small spectral red-shift and the halfwidth of a Gaussian accounting for additional spectral broadening.

*3 In ref. [2] the lifetime $\tau_{3} \approx 0.13$ ps was calculated with eq. (31). The difference between this value and $\tau_{3}^{\prime} p_{3} / N_{3} p_{1} \approx 0.26 \mathrm{ps}$ in eq. (33) results from the incorrect calculation of $\phi_{\mathrm{DF} 3}$ in ref. [2] (the incorrect equation $\phi_{\mathrm{FE}}=\phi_{\mathrm{FI}}$ was used). 
(2) If nonradiative TTA is regarded as a special case of Dexter energy transfer, then in the case of centrosymmetric molecules and at sufficiently low temperatures, no delayed fluorescence is expected at $2 E\left(T_{1,0}\right)$. Hence an observed delayed luminescence at $2 E\left(\mathrm{~T}_{1,0}\right)$ should be practically exclusively radiative TTA.

(3) In the spectra of the delayed luminescence from liquid solutions of pyrene and phenazine, the highenergy regions close to $2 E\left(\mathrm{~T}_{1,0}\right)$ are very similar to the calculated spectra of radiative TTA.

(4) If for this similarity the high-energy regions of the delayed luminescence are assigned to radiative TTA, the ratio $k_{\mathrm{r}} / k_{\mathrm{nr}}$ of the rate constants of radiative and nonradiative TTA can be determined. $k_{\mathrm{r}} / k_{\mathrm{nr}}$ is extremely small - in accord with theoretical orderof-magnitude estimates [5-7,12]: $k_{\mathrm{r}} / k_{\mathrm{nr}} \approx 5 \times 10^{-7}$ for pyrene and $k_{\mathrm{r}} / k_{\mathrm{nr}} \approx 3 \times 10^{-8}$ for phenazine.

(5) In the spectrum of the delayed luminescence from anthracene, the intensity distribution above $E\left(\mathrm{~S}_{1,0}\right)$ and below $2 E\left(\mathrm{~T}_{1,0}\right)$ can be interpreted as delayed fluorescence from $S_{1}$ vibronic states. The delayed luminescence at $2 E\left(\mathrm{~T}_{1,0}\right)$ is the only band assignable to radiative TTA.

(6) The spectrum of the delayed luminescence from 1,2-benzanthracene extends above $2 E\left(T_{1,0}\right)$, due to annihilation of vibrationally excited triplets. In this high-energy region, a clear distinction between delayed fluorescence and radiative TTA is not possible, though the latter seems to be more likely.

(7) If the spectrum of the dimol luminescence from metastable singlet oxygen is calculated in the same way as a spectrum of radiative TTA, an incorrect intensity distribution is obtained.

(8) In view of the uncertainty of the true spectrum of radiative TTA, there is still some uncertainty left about the observability of radiative TTA from aromatic compounds. Nevertheless one may safely assume that the values of the relative probability $k_{\mathrm{r}} / k_{\mathrm{nr}}$ in sections 5.1-5.3 are reliable upper bounds. If the results obtained with pyrene and phenazine are generalized, then $k_{\mathrm{r}} / k_{\mathrm{nr}} \lesssim 5 \times 10^{-7}$ is typical for compounds satisfying the conditions for the detection of radiative TTA.

(9) The spectrum of the excimer fluorescence from pyrene has no distinct origin band at temperatures $\geqslant 118 \mathrm{~K}$.

(10) From the quantum yield of the delayed $S_{n} \rightarrow S_{0}$ fluorescence and from the strength of the inhomogeneously broadened $S_{0} \rightarrow S_{n}$ absorption band, in general only a lower bound of the lifetime of $S_{n}$ can be calculated.

(11) The vibronic coupling of $S_{3}$ of 1,2-benzanthracene with lower excited states is likely to be an example of the statistical limit.

\section{Note added in proof}

After the acceptance of this paper for publication we received a belated reviewer's report. For the reader interested in the theoretical aspects of our work we take the reviewer's remarks as focal points for some additional clarifications.

(1) One may ask whether the distinction between direct radiative TTA and delayed fluorescence from a singlet state in resonance with the triplet pair is really meaningful. (The problem is similar to that of a distinction between resonance Raman scattering and resonance fluorescence.) At present our best argument for this distinction is the strong resemblance of the calculated spectrum of radiative TTA to the high-energy range of the spectrum of the delayed luminescence in the cases of pyrene and phenazine. However, on the basis of the few investigated compounds, we cannot rule out the possibility that this resemblance is fortuitous.

(2) In aromatic crystals, charge transfer excitations play an important role for triplet excitons, which have a band structure (Davydov splitting) that cannot be properly computed if the corresponding terms are neglected. In solution, similar interactions may affect both the binding energy of the pair and the transition matrix elements.

(3) In principle, in TTA the pertinent symmetry is not that of the single molecule but that of the molecular pair, which is low unless a well defined highsymmetry dimer is formed. Perturbation due to the solvent may further reduce the symmetry. Nevertheless, as fas as the overlap integral of the phosphorescence spectrum with the $T_{1} \rightarrow S_{n}$ absorption spectrum is of importance in nonradiative TTA (as assumed by us), the application of symmetry selection rules to transitions in the individual molecules may be appropriate.

(4) We interpret the origin bands of the delayed 
luminescence from pyrene, phenazine and anthracene as radiative TTA and assign the red-shift $\Delta \tilde{\nu}_{\mathrm{TT}}$ of the origin relative to the energy $2 E\left(T_{1,0}\right)$ to the triplet-triplet interaction energy. Alternatively one could interpret the origin band as delayed fluorescence and assign $\Delta \tilde{\nu}_{\mathrm{TT}}$ to a low-energy mode of the appropriate u symmetry that lifts the parity forbiddeness of the $T_{1,0}^{(u)} \rightarrow S_{n, v_{n}}^{(u)}$ transition (cf. case $B$ in section 2.2). Therefore, even if the symmetry argument is in principle accepted, it may be not decisive in making the difference between radiative TTA and delayed fluorescence from a really formed excited singlet state.

\section{Acknowledgement}

We thank Professor A. Weller for support of this work, Dr. D. Klemp for the measurement of the phosphorescence spectrum of pyrene and for the repetition of the measurement of the delayed luminescence from phenazine, Dr. M.F. Rodríguez Prieto for the measurement of excimer fluorescence spectra of pyrene, Mr. A. Ruth for the measurement of the absorption spectrum of phenazine, $\mathrm{Mr}$. $\mathrm{H}$. Lesche for the purification of substances and the preparation of samples, and Dr. R. Schmidt (University of Frankfurt) for the communication of unpublished results.

\section{References}

[1] C.A. Parker, Photoluminescence from Solutions (Elsevier, Amsterdam, 1968).

[2] B. Nicke], Helv. Chim. Acta 61 (1978) 198.

[3] H. Sternlicht, G.C. Nieman and G.W. Robinson, J. Chem. Phys. 38 (1963) 1326.

[4] R.E. Merrifield, Pure Appl. Chem. 27 (1971) 481.

[5] R.S. Knox and C.E. Swenberg, J. Chem. Phys. 44 (1966) 2577.

[6] J. Jortner and S.A. Rice, J. Chem. Phys. 44 (1966) 3364.

[7] S.A. Rice and J. Jortner, in: The Organic Solid State, Vol. 3. Comments on the Theory of Exciton States in Molecular Crystals, eds. D. Fox, M.M. Labes and A. Weissberger (Interscience, New York, 1967) pp. $377 \mathrm{ff}$.

[8] C. Mavroyannis, Phys. Rev. B 1 (1970) 2706.

[9] V. Ern, H. Bouchriha, M. Bisceglia, S. Arnold and M. Schott, Phys. Rev. B 8 ( 1973 ) 6038.

[10] J.W. Ellis and H.O. Kneser, Z. Physik. 86 (1933) 583.

[ 11 ] S.J. Arnold, E.A. Ogryzlo and H. Witzke, J. Chem. Phys. 40 (1964) 1769.

[12] C.E. Swenberg, J. Chem. Phys. 51 (1969) 1753.
[13] M. Schott, private communication.

[14] B. Nickel, Chem. Phys. Letters 68 (1979) 17.

[15]D. Klemp, Dissertation, Universität Göttingen, FRG (1985).

[16] Th. Förster, Fluoreszenz Organischer Verbindungen (Vandenhoeck \& Rupprecht, Göttingen, 1951) \$12.

[17] J. Jortner, S.A. Rice, J.L. Katz and S.-I. Choi, J. Chem. Phys. 42 (1965) 309.

[18] P.R. Salvi, P. Foggi and E. Castellucci, Chem. Phys. Letters 98 (1983) 206.

[19] A. Nitzan, J. Jortner and P.M. Rentzepis, Proc. Roy. Soc. A 327 (1972) 367.

[20] C.J. Ho, R.J. Babbitt and M.R. Topp, J. Phys. Chem. 91 (1987) 5599.

[21] B. Nickel and J. Hertzberg, Chem. Phys. 132 (1989) 219.

[22] A. Bree and V.V.B. Vilkos, Spectrochim. Acta 27 A (1971) 2333.

[23] E.A. Mangel and M.R. Topp, J. Phys. Chem. 90 (1986) 802.

[24] D.L. Dexter, J. Chem. Phys. 21 (1953) 836.

[25] J. Hertzberg and B. Nickel, Chem. Phys. 132 (1989) 235.

[26] D. Wyrsch and H. Labhart, Chem. Phys. Letters 8 (1971) 217.

[27] H. Labhart and D. Wyrsch, Chem. Phys. Letters 12 (1971) 378.

[28] P.R. Butler and M.J. Pilling, J. Chem. Soc. Faraday 1173 (1977) 886.

[29] C.A. Parker, Proc. Roy. Soc. A 276 (1963) 125.

[30] B. Nickel and G. Roden, Chem. Phys. 66 (1982) 365.

[31] D. Klemp and B. Nickel, Chem. Phys. 78 (1983) 17.

[32] B. Nickel and G. Roden, Ber. Bunsenges. Physik. Chem. 81 (1977) 281.

[33] B. Nickel and M.F. Rodríguez Prieto, Z. Physik. Chem. NF 150 (1986) 31.

[34] D. Klemp and B. Nickel, Chem. Phys. Letters 130 (1986) 493.

[35] W. Heinzelmann and H. Labhart, Chem. Phys. Letters 4 (1969) 20.

[36] J.B. Birks, Photophysics of Aromatic Molecules (WileyInterscience, New York, 1970).

[37] B. Nickel and G. Roden, Chem. Phys. Letters 71 (1980) 238.

[38] D.L. Narva and D.S. McClure, Chem. Phys. 56 (1981) 167.

[39] A. Grabowska and B. Pakuta, Photochem. Photobiol. 9 (1969) 339.

[40] Y. Hirata and I. Tanaka, Chem. Phys. Letters 43 (1976) 568.

[41] H.-B. Lin and M. Topp, Chem. Phys. 36 (1979) 365.

[42] S.F. Dobrinevskii, S.A. Tikhomirov and G.B. Tolstorozhev, Opt. i Spekroskopiya 66 (1989) 293.

[43] I. Carmichael and G.L. Hug, J. Phys. Chem. Ref. Data 15 (1986) 1.

[44] S. Voelker and R.M. Macfarlane, Chem. Phys. Letters 61 (1979) 421.

[45] W.H. Hesselink and D.A. Wiersma, J. Chem. Phys. 74 (1981) 886.

[46] P.M. Rentzepis and V.E. Bondybey, J. Chem. Phys. 80 (1984) 4727. 
[47] W. Kaiser, Ber. Bunsenges. Physik. Chem. 89 (1985) 213.

[48] W. Kaiser and A. Seilmeier, Ber. Bunsenges. Physik. Chem. 91 (1987) 1201.

[49] B. Dick, private communication.

[50] T. Tamm and P. Saari, Chem. Phys. 40 (1979) 311.

[51] A. Freiberg, T. Tamm and K. Timpmann, Laser Chem. 3 (1983) 249.

[52] N.H. Gottfried and W. Kaiser, Chem. Phys. Letters 101 (1983) 331.

[53] M. v. Löwis of Menar, J. Bendig and M. Siegmund, J. Prakt. Chem. 325 (1983) 75.

[54] W.R. Lambert, P.M. Felker, J.A. Syage and A.H. Zewail, J. Chem. Phys. 81 (1984) 2195.

[55] A. Amirav, C. Horwitz and J. Jortner, J. Chem. Phys. 88 (1988) 3092.

[56] K.H. Grellmann and H.-G. Scholz, Chem. Phys. Letters 62 (1979) 64.

[57] P.R. Salvi and G. Marconi, J. Chem. Phys. 84 (1986) 2542.

[58] R.G. Bennett and P.J. McCartin, J. Chem. Phys. 44 (1966) 1969.

[59] E.C. Lim, J.D. Laposa and J.M.H. Yu, J. Mol. Spectry. 19 (1966) 412.

[60] B. Nickel, Chem. Phys. Letters 27 (1974) 84.

[61 ] C.E. Easterley, L.G. Christophorou and J.G. Carter, J. Chem. Soc. Faraday II 69 (1973) 471.

[62] K. Gustav and G. Seydenschwanz, Z. Physik. Chem. 268 (1987) 369.

[63] H.-B. Lin and M.R. Topp, Chem. Phys. Letters 48 (1977) 251.

[64] V.L. Bogdanov and V.P. Klochkov, Opt. i Spekroskopiya 45 (1978) 95.

[65] V.L. Bogdanov and V.P. Klochkov, Opt. i Spektroskopiya 52 (1982) 71 .

[66] V.L. Bogdanov, Opt. i Spektroskopiya 56 (1984) 441.

[67] V.L. Ermolaev and V.A. Lyubimtsev, Acta Phys. Polon. A 71 (1987) 731.

[68] V.A. Lyubimtsev and V.L. Ermolaev, Opt. i Spektroskopiya 64 (1988) 1030.

[69] B. Nickel, unpublished results.

[70] K.I. Salokhiddinov, B.M. Dzhagarov, I.M. Byteva and G.P. Gurinovich, Chem. Phys. Letters 76 (1980) 85
[71] R. Schmidt, Habilitationsschrift, Universität Frankfurt, FRG (1989).

[72] A.U. Khan and M. Kasha, J. Chem. Phys. 39 (1963) 2105.

[73] J.S. Arnold, R.J. Browne and E.A. Ogryzlo, Photochem. Photobiol. 4 (1965) 963.

[74] E.W. Gray and E.A. Ogryzlo, Chem. Phys. Letters 3 (1969) 658.

[75] R. Protz and M. Maier, J. Chem. Phys. 73 (1980) 5464.

[76] P.M. Borrell, P. Borrell and K.R. Grant, J.Chem. Soc. Faraday II 76 (1980) 1442.

[77] C. Dijkgraaf and G.J. Hoijtink, Tetrahedron 19 (1963) Suppl. 2, 179.

[78] S.P. McGlynn, T. Azumi and M. Kasha, J. Chem. Phys. 40 (1964) 507.

[79] D.F. Evans, J. Chem. Soc. (London) (1957) 1351.

[80] S.P. McGlynn, T. Azumi and M. Kinoshita, Molecular Spectroscopy of the Triplet State (Prentice-Hall, Englewood Cliffs, 1969) ch. 8.

[81 ] C.A. Parker, Chem. Phys. Letters 6 (1970) 516.

[82] F. Tfibel and L. Lindqvist, Chem. Phys. 10 (1975) 471.

[83] D.K.K. Liu and L.R. Faulkner, J. Am. Chem. Soc. 100 (1978) 2635.

[84] J. Saltiel, G.R. Marchand, W.K. Smothers, S.A. Stout and J.L. Charlton, J. Am. Chem. Soc. 103 (1981) 7159.

[85] N.A. Borisevich, A.V. Dorokhin and A.A. Sukhodola, Opt. i Spektroskopiya 59 (1985) 1327

[86] B. Dick and B. Nickel, Chem. Phys. 78 (1983) 1.

[87] D.A. Antheunis, B.J. Botter, J. Schmidt, P.J.F. Verbeek and J.H. van der Waals, Chem. Phys. Letters 36 (1975) 225.

[88 ] J. Spichtig, H. Bulska and H. Labhart, Chem. Phys. 15 (1976) 279.

[89] D.F. Evans, in: Optische Anregung Organischer Systeme, 2. Internationales Farbensymposium 1964 (Verlag Chemie, Weinheim, 1966 ) p. 590.

[90] S.J. Strickler and R.A. Berg, J. Chem. Phys. 37 (1962) 814.

[91] A. Amirav, M. Sonnenschein and J. Jortner, J. Phys. Chem. 88 (1984) 5593.

[92] B. Dick and B. Nickel, Chem. Phys. 110 (1986) 131.

[93] M. Lindrum and B. Nickel, Chem. Phys. 144 (1990) 129. 